\title{
Sequencing depth and genotype quality: accuracy and breeding operation considerations for genomic selection applications in autopolyploid crops
}

\author{
Dorcus C. Gemenet ${ }^{1,4}\left(\right.$ D $\cdot$ Hannele Lindqvist-Kreuze ${ }^{2} \cdot$ Bert De Boeck $^{2} \cdot$ Guilherme da Silva Pereira $^{3,5} \cdot$ \\ Marcelo Mollinari ${ }^{3} \cdot$ Zhao-Bang Zeng $^{3} \cdot$ G. Craig Yencho ${ }^{3} \cdot$ Hugo Campos $^{2}$
}

Received: 1 March 2020 / Accepted: 21 August 2020 / Published online: 2 September 2020

(c) The Author(s) 2020

Key message Polypoid crop breeders can balance resources between density and sequencing depth, dosage information and fewer highly informative SNPs recommended, non-additive models and QTL advantages on prediction dependent on trait architecture.

Abstract The autopolyploid nature of potato and sweetpotato ensures a wide range of meiotic configurations and linkage phases leading to complex gene-action and pose problems in genotype data quality and genomic selection analyses. We used a 315-progeny biparental $F_{1}$ population of hexaploid sweetpotato and a diversity panel of 380 tetraploid potato, genotyped using different platforms to answer the following questions: (i) do polyploid crop breeders need to invest more for additional sequencing depth? (ii) how many markers are required to make selection decisions? (iii) does considering non-additive genetic effects improve predictive ability (PA)? (iv) does considering dosage or quantitative trait loci (QTL) offer significant improvement to PA? Our results show that only a small number of highly informative single nucleotide polymorphisms (SNPs; $\leq 1000)$ are adequate for prediction in the type of populations we analyzed. We also show that considering dosage information and models considering only additive effects had the best PA for most traits, while the comparative advantage of considering non-additive genetic effects and including known QTL in the predictive model depended on trait architecture. We conclude that genomic selection can help accelerate the rate of genetic gains in potato and sweetpotato. However, application of genomic selection should be considered as part of optimizing the entire breeding program. Additionally, since the predictions in the current study are based on single populations, further studies on the effects of haplotype structure and inheritance on PA should be studied in actual multi-generation breeding populations.

\section{Introduction}

Communicated by Herman J. van Eck.

Electronic supplementary material The online version of this article (https://doi.org/10.1007/s00122-020-03673-2) contains supplementary material, which is available to authorized users.

\section{Dorcus C. Gemenet}

d.gemenet@cgiar.org

1 International Potato Center, ILRI Campus, P.O. Box 25171-00603, Nairobi, Kenya

2 International Potato Center, Av. La Molina 1895, Lima, Peru

3 North Carolina State University, Raleigh, NC 27695, USA

4 Present Address: CGIAR Excellence in Breeding Platform, International Maize and Wheat Improvement Center (CIMMYT), ICRAF Campus, 1041-00621 Nairobi, Kenya

5 Present Address: International Potato Center, Nairobi, Kenya
Phenotyping under recurrent selection has been an important approach for variety development in plant breeding, with substantial success to date. However, this process may take a long time for most crops, particularly for clonally propagated crops (Slater et al. 2016). For example, in potato, it typically takes an entire year to develop enough tubers from botanical seed obtained from crossing nurseries, for experimental trial purposes. This is followed by at least 2 years of field evaluation for qualitative traits, with evaluation for most quantitative traits in replicated multi-environment trials beginning in around year four (Endelman et al. 2018). The same can be said for sweetpotato, although cycle times in sweetpotato are shorter by about a year due to the fact that the crop can be vegetatively propagated via stem cuttings (Grüneberg et al. 2009). This represents a stark contrast with what can be achieved in cereal and legume crops, where up 
to 6 generations can be raised within a calendar year (Watson et al. 2018), or in private corn breeding programs based in the USA and Europe which can raise multiple generations per year through the coordinated use of winter nurseries located in both hemispheres such as USA, Puerto Rico, Hawaii and Chile. This therefore implies that the estimation of parental value based on genetic designs and phenotypic evaluation in potato and sweetpotato increases the selection cycle time, thereby reducing the rate of genetic gains and the speed of delivery of superior, novel genetics to farmers.

The use of genetic markers for selection offers potential to reduce the breeding cycle time as selection can be done at an earlier stage. Previously proposed methods have involved identifying quantitative trait loci (QTL) via QTL mapping and genome-wide association studies (GWAS), but they have had little practical application in the actual development of new cultivars through plant breeding to date, especially for complex quantitative traits, since identifying the causal genes underlying QTL needed to make their application practical is costly (Xu and Crouch 2008). Genomic selection (GS) offers the ability to select parents within a shorter interval and increase selection intensity by predicting untested genotypes earlier while enhancing larger starting genetic variation. This approach uses genome-wide marker data to predict the performance of untested genotypes and estimate their breeding values (genomic estimated breeding values; GEBVs), based on a genotyped and phenotyped training population (Meuwissen et al. 2001). Genomic selection is emerging as the approach of choice to circumvent the limitations associated with use of QTL for marker-assisted selection and to improve the efficiency of phenotypic selection (Bernal-Vasquez et al. 2014). Good genetic progress can be made using GS, as long as factors that affect its predictive ability (PA), i.e., the correlation between phenotypic best linear unbiased estimators (BLUPs) and GEBVs, are well understood. These include trait architecture, the size of the training population, the relationship between the training and validation populations, heritability of the trait, the quality of phenotypic efforts, the level of linkage disequilibrium (LD), marker density, environmental variances and covariance among traits (Covarrubias-Pazaran et al. 2018).

The application of GS is taking shape in plant breeding with more and more crops exploring its utility (Spindel et al. 2016; Wang et al. 2018; Endelman et al. 2018; CovarrubiasPazaran et al. 2018; Faville et al. 2018; Nyine et al. 2018; Bhandari et al. 2019). For crops like rice and wheat that are normally self-pollinated and have a high incidence of higheffect QTL (Spindel et al. 2016), faster success is expected from applying GS as prediction accuracy depends primarily on the factors listed above. However, breeders of autopolyploid, clonally propagated crops like potato and sweetpotato, which are normally heterogeneous and heterozygous, have to ask themselves additional questions and identify trade-off points that enhance the success of GS-assisted breeding (Slater et al. 2016; Endelman et al. 2018). Potato and sweetpotato present a wide range of meiotic configurations and linkage phases (Mollinari et al. 2020). In addition to causing complex gene-action effects, allelic and configuration diversity have consequences on genotyping and genotype data quality, which consequently affects downstream analysis for quantitative-genetic parameters required to make high-quality breeding decisions. Genotyping-by-sequencing (GBS) has currently become a genotyping method of choice in plant breeding (Poland and Rife 2012), but it is also prone to genotyping errors and a high level of missingness at low depth of sequencing, while high sequencing depth has additional cost implications. Data from polyploid crops are more prone to low-quality genotype calls at low sequencing depth when compared to diploid crops, because of uncertain allele dosages and possibility of non-random inheritance of alleles such as in preferential pairing or double reduction (Blischak et al. 2016, 2018).

Public sector breeding programs like those conducted in centers which are part of the Consultative Group on International Agricultural Research (CGIAR), and in the individual National Agricultural Research Systems (NARS) existing in many countries, are currently undergoing breeding program optimization efforts in order to keep up with the challenges of climate change and population increase (Cobb et al. 2019). Application of GS is one such tool for breeding program optimization. In order to develop GS tools to make more effective breeding efforts in autopolyploid crops such as potato and sweetpotato, we have taken a practical perspective within a plant breeding setting to address several pertinent questions related to application of GS in autopolyploids. We used real data sets from a 380 training-panel made up of advanced tetraploid potato clones and a 315-fullsib family $\left(F_{1}\right)$ of hexaploid sweetpotato, both developed by the International Potato Center (CIP) and genotyped using different platforms, to address the following questions: (i) do polyploid crop breeders need to invest more resources for additional sequencing depth? (ii) how many genetic markers are required to make selection decisions? (iii) does the consideration of non-additive genetic effects add value to predictive ability (PA) to enhance genetic gains either for population improvement or product development in polyploid crops? (iv) given the multiple alleles at loci with diverse meiotic configurations and linkage phases, does considering dosage, haplotypic or QTL effects offer significant improvement to PA to enhance genetic advances? We also discuss other factors that need to be considered while adopting GS as a decision support tool in an optimized breeding program. 


\section{Materials and methods}

\section{Genetic materials and phenotyping}

\section{Sweetpotato biparental population}

A wide genetic variability exists in sweetpotato in terms of yield, nutritional content and culinary aspects, abiotic stress tolerance, biotic stress tolerance, among other attributes (Low et al. 2017). Introgression of high $\beta$-carotene content into locally adapted varieties is a major breeding objective especially in sub-Saharan Africa where vitamin A deficiency is prevalent. A 315-progeny full-sib family $\left(F_{1}\right)$ was developed by crossing a US-bred high $\beta$-carotene variety, 'Beauregard,' with an adapted, locally preferred, starchy, low $\beta$-carotene landrace variety, 'Tanzania,' at CIP_-Peru. These two parents differ in additional traits of interest, and the population will henceforth be referred to as the BT population. The population was evaluated in six environments of Peru, for various quality-related and yield-related traits, between 2016 and 2017. The design was an $80 \times 4 \alpha$-lattice with two-three replications, depending on location. The information about these trials is further described in the publications by Gemenet et al. (2020) and Pereira et al. (2020), and summaries of locations and experimental designs attached as Online Resource 1. Additionally, flesh color was evaluated in six environments of Uganda. The design was $80 \times 4$ $\alpha$-lattice with three replications per location, in a $4.8 \mathrm{~m}^{2}$ plot size, with three locations over two years, as further described by Gemenet et al. (2020). The quality-related traits measured in the BT population include: dry matter (DM) content, measured as a percentage of the laboratory dried samples divided by the initial fresh weight of $100 \mathrm{~g}$; Starch and $\beta$-carotene (BC) content, estimated using near-infrared reflectance spectroscopy (NIRS) and flesh color (FC), measured using internal color scales developed by CIP and partners. All quality-related traits were measured in Peru, but only flesh color was measured in Uganda (FC_U). Data are further described in Gemenet et al. (2020). For yield-related traits, total number of storage roots (TNR), number of commercial storage roots (NOCR), weight of total storage roots (RYTHA), weight of commercial storage roots (CYTHA) and total weight of foliage (FYTHA), were measured in the six experiments of Peru only. Data are further described in Pereira et al. (2020). Trait abbreviations are further defined in Table 1.

The quality-related traits were analyzed by fitting the following linear mixed model in ASREML:

$y_{i j k l}=\mu+g_{i}+e_{l}+r_{k(l)}+b_{j k(l)}+\left(g e_{i}\right)_{l}+\varepsilon_{i j k(l)}$

where $y_{i j k l}=$ the vector of phenotypes of genotype $i$ in block $j$ within replicate $k$ of environment $l, \mu=$ population mean, $g_{i}=$ the fixed treatment (genotype) effect, $e_{l}=$ the random effect of environment $l, r_{k(l)}=$ random effect of replicate $k$ in environment $l, b_{j k(l)}=$ random effect of block $j$ within replicate $k$ of environment $l,\left(g e_{i}\right)_{l}=$ random effect of genotype $i$ in environment $1(1=1, . ., L), \varepsilon_{i j k(l)}=$ random error of the residuals, assuming $e_{l} \sim N\left(0, \sigma_{e}^{2}\right), \quad r_{k(l)} \sim N\left(0, \sigma_{r(l)}^{2}\right)$, $b_{j k(l)} \sim N\left(0, \sigma_{b(l)}^{2}\right), g e_{i} \sim N(0, \Sigma)$ with $\sum=$ variance-covariance matrix of the genotypes across $L=5$ environments, allowing for heterogeneity of genetic variances and covariances across environments. The best fitting model was
Table 1 Trait abbreviations and their description in sweetpotato and potato as used in the current study

\begin{tabular}{lll}
\hline Crop & Trait abbreviation & Trait description \\
\hline Sweetpotato & DM & Dry matter content \\
& Starch & Starch content \\
BC & Beta-carotene \\
FC_P & Flesh color in Peru \\
FC-U & Flesh color in Uganda \\
NOCR & \# Commercial storage roots \\
TNR & \# Total storage roots \\
CYTHA & Commercial storage root weight \\
RYTHA & Total storage root weigh \\
FYTHA & Total foliage yield weight \\
LB2014_O & Late blight in 2014 in Oxapampa, Peru \\
LB2016_Y & Late blight 2016 in Yunnan, China \\
PVY_L & Potato virus Y in Lima, Peru \\
AYP_K & Average yield per plant in Kunming, China \\
WMT_K & Weight of marketable tubers in Kunming, China \\
TTW16_Ica & Total tuber weight in 2016 in Ica-Peru \\
TTW16_HLJ & Total tuber weight in 2016 in Heilongjiang, China \\
&
\end{tabular}


chosen by Akaike's information criterion (AIC) and differed slightly for the different traits. For DM, Starch and FC_U, a factor analytic model (Piepho 1998) of order 1 was used, and for $\mathrm{BC}$ a factor analytic model of order 2, to model the variance covariance matrix $\sum$. For FC, an unstructured variance-covariance matrix $\sum$ was used in the final model. The random error of residuals was assumed as $\varepsilon_{i j k(l)} \sim N\left(0, \sigma_{l}^{2}\right)$ (Gemenet et al. 2020).

The yield-related traits were also analyzed with linear mixed models as described by Pereira et al. (2020) using restricted maximum likelihood (REML) in GENSTAT 14 as:

$y_{i j k l}=\mu+g_{i}+e_{l}+r_{k(l)}+b_{j k l}+g e_{i l}+\varepsilon_{i j k l}$

where $y_{i j k l}=$ the vector of phenotypes as above, $\mu=$ population mean, $g_{i}=$ the fixed treatment (genotype) effect, $e_{l}=$ fixed effect of environment $l, r_{k(l)}=$ fixed effect of replication $k$ in environment $l, b_{j k l}=$ random effect of block $j$ within replication $k$ in environment $l ; b_{j k l} \sim N\left(0, \sigma_{b}^{2}\right), g e_{i l}=$ the fixed interaction effect of genotype $i$ and environment $l$, and $\varepsilon_{i j k l} \sim N\left(0, \sigma^{2}\right)$ is the random residual error. The two data classes (quality-related traits and yield-related traits) were analyzed using different methods because the two analysts made different assumptions regarding variance components and genotype-by-environment interaction. The models for yield-related traits assumed compound symmetry and fixed correlation among environments, which may be less realistic in estimating the covariance structure of the different traits. This could lead to poor estimates of standard errors of differences between some means and hence some difference in adjusted means. However, such differences are not expected to significantly affect the findings from further analyses using these adjusted means in the current study. The best linear unbiased estimators (BLUEs) as obtained by fitting the above models to the experimental data with genotypes as fixed were then used to estimate GEBVs.

\section{Potato trait observation network population}

A 380-genotype panel made up of advanced clones from the potato breeding program and representing all breeding populations at CIP was assembled for a trait observation network (TON) in Peru, China and Ethiopia. Henceforth, we shall refer to this population as the TON panel. The evaluation of the panel was carried out in diverse agro-ecological zones, and in subsets of genotypes subject to participating NARS' partner capacity and/or ability to produce enough minitubers for experimentation. The experimental sites, experimental designs and the number of genotypes evaluated per experiment are summarized in Table 2. The TON panel was evaluated for maturity (bulking) by tuber characteristics at three harvest dates where average yield per plant (kg; AYP) and weight of marketable tubers per plant (kg; WMT) were measured. Additionally, mature tuber weight was evaluated by measuring total tuber weight per plant (TTW; $\mathrm{kg}$ ). In Peru, TTW was measured as the average total tuber weight across three drought-related treatments: terminal drought (irrigation stopped at flowering until harvest; TTW16_TD), recovery (partially irrigated after drought stress; TTW16_ REC) and fully irrigated (normally irrigated throughout the growth period; TTW16_NI), while random drought was used in China, with no controlled treatments. Resistance to potato virus Y (PVY) was evaluated after infection with virulent vectors and susceptible spreader rows using standard protocols at CIP, while late blight resistance (LB) was evaluated by growing the population in endemic disease

Table 2 Locations, designs and traits measured in the trait observation network (TON) panel of potato

\begin{tabular}{|c|c|c|c|c|}
\hline Country & \multicolumn{2}{|l|}{ Location } & & Agroecology \\
\hline \multirow[t]{3}{*}{ Peru } & \multicolumn{2}{|c|}{ Lima, La Molina $12.0820^{\circ} \mathrm{S}, 76.9282^{\circ} \mathrm{W}$} & & \multirow[t]{2}{*}{ Lowland sub-tropics } \\
\hline & \multirow{2}{*}{\multicolumn{2}{|c|}{$\begin{array}{l}\text { Ica, Ica } 14.0755^{\circ} \mathrm{S}, 75.7342^{\circ} \mathrm{W} \\
\text { Pasco, Oxapampa } 10.5853^{\circ} \mathrm{S}, 75.4053^{\circ} \mathrm{W}\end{array}$}} & & \\
\hline & & & & Highland tropics \\
\hline \multirow[t]{3}{*}{ China } & \multicolumn{2}{|c|}{ Yunnan, Kunming $24.8801^{\circ} \mathrm{N}, 102.8329^{\circ} \mathrm{E}$} & & \\
\hline & \multicolumn{2}{|c|}{ Mixed agriculture systems, lowland \& highland } & & \multirow[b]{2}{*}{ Temperate (long day) } \\
\hline & \multicolumn{2}{|c|}{ Heilongjiang, Harbin $45.8038^{\circ} \mathrm{N}, 126.5350^{\circ} \mathrm{E}$} & & \\
\hline Trait group & Trait & Location, country, year & Trial design & \#Genotype \\
\hline \multirow[t]{2}{*}{ Late blight resistance } & LB2014_O & Oxapampa, Peru, 2014 & RCBD & 241 \\
\hline & LB2016_Y & Yunnan, China, 2016 & RCBD & 336 \\
\hline Virus resistance & PVY_L & Lima, Peru, 2016-2018 & RCBD & 341 \\
\hline \multirow[t]{2}{*}{ Bulking-based maturity } & AYP_K & Kunming, China, 2016 & RCBD & 317 \\
\hline & WMT_K & Kunming, China, 2016 & RCBD & 317 \\
\hline \multirow[t]{2}{*}{ Mature tuber weight } & TTW16_Ica & Ica, Peru, 2016 & Augmented & 269 \\
\hline & TTW16_HLJ & Heilongjiang, China, 2016 & Augmented & 300 \\
\hline
\end{tabular}


pressure and scored using standard protocols at CIP. Trait abbreviations are defined in Table 1.

Unlike in sweetpotato where phenotype and genotype data were balanced across experiments, $(292+$ Parents for DArTSeq and 315 + parents for GBSpoly), the potato experiments were unbalanced in terms of experimental genotypes. For the purposes of this study, we only selected the locations with the highest training population per trait. Consequently, we used AYP from Kunming (China; AYP_K), WMT from Kunming (China; WMT_K), LB from Oxapampa (Peru; LB2014_O), LB from Yunnan (China; LB2016_Y), PVY from Lima (Peru; PVY_L), TTW averaged across three treatments of 2016 in Ica (Peru; TTW16_Ica) and TTW in 2016 from Heilongjiang (China; TTW16_HLJ), all having number of genotypes indicated in Table 2. The experiments were analyzed as single trials, depending on the experimental design used as summarized in Table 2. A linear mixed model, taking into account the respective experimental design, was fitted to the phenotypic data. For those traits with different treatments like TTW in Peru, the joint adjusted means were additionally obtained across all treatments by fitting a linear mixed model. Genotype was considered as a fixed effect in these mixed models, so that BLUEs for the genotypic means were obtained for each trait and used to predict GEBVs.

\section{Genotyping and variant calling}

The full 315-progeny of the BT (sweetpotato) population was genotyped together with the parents using an optimized protocol for hexaploid sweetpotato, 'GBSpoly' at North Carolina State University (NCSU). Additionally, a subsample of 292-progeny and the two parents of the BT population were genotyped by DArTSeq ${ }^{\mathrm{TM}}$ in Australia, under the collaboration between the Integrated Genotyping Service and Support (IGSS) platform at the Biosciences east and central Africa (BecA) hub in Nairobi, Kenya and DArT. The 380 genotypes of the TON population (potato) were genotyped by GBS at Cornell University.

\section{DArTSeq $^{\text {TM }}$ for Sweetpotato}

DArTseq ${ }^{\mathrm{TM}}$ represents a combination of DArT complexity reduction methods and next-generation sequencing platforms (Kilian et al. 2012; Courtois et al. 2013; Raman et al. 2014; Cruz et al. 2013). Therefore, DArTseq ${ }^{\mathrm{TM}}$ represents a new implementation of sequencing complexity reduced representations (Altshuler et al. 2000) and more recent applications of this concept on the next-generation sequencing platforms (Baird et al. 2008; Elshire et al. 2011). Similar to previous DArT methods based on array hybridizations, the technology is optimized for each organism and application by selecting the most appropriate complexity reduction method (both the size of the representation and the fraction of a genome selected for assays). Four methods of complexity reduction were tested in sweetpotato (data not presented), and the PstI-MseI method was selected. DNA samples were processed in digestion/ligation reactions principally as per Kilian et al. (2012) but replacing a single PstI-compatible adaptor with two different adaptors corresponding to two different restriction enzyme (RE) overhangs. The PstI-compatible adapter was designed to include Illumina flowcell attachment sequence, primer sequence and 'staggered,' varying length barcode region, similar to the sequence reported by Elshire et al. (2011). This reverse adapter contained a flowcell attachment region and a MseI-compatible overhang sequence. Only 'mixed fragments' (PstI-MseI) were effectively amplified in 30 rounds of PCR using the following reaction conditions: (i) $94^{\circ} \mathrm{C}$ for $1 \mathrm{~min}$, (ii) 30 cycles of: $94{ }^{\circ} \mathrm{C}$ for $20 \mathrm{~s}, 58^{\circ} \mathrm{C}$ for $30 \mathrm{~s}, 72{ }^{\circ} \mathrm{C}$ for $45 \mathrm{~s}$ and (iii) $72{ }^{\circ} \mathrm{C}$ for $7 \mathrm{~min}$. After PCR, equimolar amounts of amplification products from each sample of the 96-well microtiter plate were bulked and applied to c-Bot (Illumina) bridge PCR followed by sequencing on Illumina Hiseq 2000. The sequencing (single read) was run for 77 cycles. Sequences generated from each lane were processed using proprietary DArT analytical pipelines. In the primary pipeline, the FastQ files were first processed to filter away poor-quality sequences, applying more stringent selection criteria to the barcode region compared to the rest of the sequence. This was to ensure reliability in the assignments of the sequences to specific samples carried in the 'barcode split' step. Approximately 2,000,000 sequences per barcode/sample were identified and used in marker calling. Finally, identical sequences were collapsed into 'fastqcoll files.' The fastqcoll files were 'groomed' using DArT PL's proprietary algorithm which corrects low-quality base from singleton tag into a correct base using collapsed tags with multiple members as a template. The 'groomed' fastqcoll files were used in the secondary pipeline for DArT PL's proprietary SNP and SilicoDArT (presence/absence of restriction fragments in representation) calling algorithms (DArTsoft14). For SNP calling, all tags from all libraries included in the DArTsoft14 analysis were clustered using DArT PL's C++algorithm at the threshold distance of 3 , followed by parsing of the clusters into separate SNP loci using a range of technical parameters, especially the balance of read counts for the allelic pairs. Additional selection criteria were added to the algorithm based on analysis of approximately 1000 controlled cross populations. Testing a range of tag counts parameters facilitated selection of true allelic variants from paralogous sequences. In addition, multiple samples were processed from DNA to allelic calls as technical replicates and scoring consistency was used as the main selection criteria for high-quality/low error rate markers. Calling quality was assured by high average read depth per locus $(>30 \mathrm{X})$. The SNPs were coded as 
$0=\mathrm{AA}, 1=\mathrm{BB}, 2=\mathrm{AB}$ and '-'= Missing. The sequences were not aligned to a reference genome because by the time of genotyping, the diploid references (Wu et al. 2018) had not been published.

\section{GBSPoly® for Sweetpotato}

GBSpoly is an optimized protocol for hexaploid sweetpotato developed at NCSU as part of a project focusing on developing genomic tools for sweetpotato improvement. The DNA was checked for quality on $1 \%$ agarose gel and quantified based on the PicoGreen florescence-based assay and the concentration was normalized to $50 \mathrm{ng} / \mu \mathrm{l}$. Initially, several optimization efforts regarding restriction enzyme pairing were carried out (data not shown) and CviAII-TseI was selected to be the best combination for hexaploid sweetpotato. Therefore, $1 \mu \mathrm{g}$ of DNA was double-digested using five units of $C$ viAII for three hours at $25^{\circ} \mathrm{C}$ followed by digestion with TseI for another three hours at $65^{\circ} \mathrm{C}$. A new England Biolabs (NEB) CutSmart buffer was used to make up a total volume of $30 \mu \mathrm{l}$. Purification of the digested samples was done using AMPure XP magnetic beads from ThermoFisher ${ }^{\mathrm{TM}}$ and quantified with PicoGreen assay. Barcodes were designed to account for substitution and indel errors and had an 8-bp buffer sequence to ensure that the barcode lay within high-quality base call regions of the sequence reads. Additional double digests on 64-plex pooled samples, purification and size selection steps were carried out as described by Wadl et al. (2018) before performing $125 \mathrm{bp}$ single-end sequencing on a total of 40 sequencing lanes ( 8 lanes for each of the 5 libraries) of the Illumina HiSeq 2500 platform. The resultant FastQ files were aligned to reference genomes of two wild relatives of sweetpotato, Ipomoea trifida and Ipomoea triloba (Wu et al. 2018), and variant calling done using the GBSapp pipeline as described by Wadl et al. (2018). The SNPs were coded according to the dosage of the alternative allele as $0=\mathrm{AAAAAA}, 1=\mathrm{AAAAAB}$, $2=\mathrm{AAAABB}, 3=\mathrm{AAABBB}, 4=\mathrm{AABBBB}, 5=\mathrm{ABBBBB}$, $6=\mathrm{BBBBBB}$. The variant calling process is summarized in Online Resource 2.

\section{GBSCornell for potato}

The 380-genotype TON panel was genotyped by Cornell University using GBS in 2015. The DNA was digested with EcoT221 restriction enzyme, and 48-plex libraries were prepared for sequencing, using customized GBS protocols at Cornell. The resultant FastQ files were quality controlled and variant calling done using GATK HaplotypeCaller option (Poplin et al. 2017), disabling the duplicate read filter (this is recommended for GBS data) and using the joint genotyping -ERC GVCF mode, as further described in Lindqvist-Kreuze et al. (2020). The reads were aligned to the potato genome reference sequenced from $S$. tuberosum group Phureja, line DM1-3 516 R44, a doubled monoploid (DM) via anther culture by the potato genome sequencing consortium (PGSC). Version PGSC_DM_v4.03 of the reference genome was used in alignment. The barcodes were removed using stacks, and the ends were trimmed using trim-galore, followed by mapping to the reference using BWA. Resultant SAM files were processed using samtools and variants called using GATK Haplotype caller, targeting biallelic SNPs only. The SNPs were coded according to the dosage of the alternative allele as $0=\mathrm{AAAA}, 1=\mathrm{AAAB}, 2=\mathrm{AABB}, 3=\mathrm{ABBB}$ and $4=\mathrm{BBBB}$. The SNP filtering was done using bcftools allowing only for those SNPs with MAF of $\geq 3 \%$, call rate of $\geq 70 \%$, average genotype quality (GQ) $\geq 30$ and minimum read depth (DP) $\geq 16$ (Lindqvist-Kreuze et al. 2020).

Although estimating allele frequencies in polyploids may encounter many challenges as explained by De Silva et al. (2005), allele frequencies for the polyploid data ('GBSpoly' for sweetpotato and 'GBSCornell' for potato) in the current study were estimated by counting the number of alleles in each dosage-based genotype, since quantitative genotyping was used for both methods.

\section{Model comparison for predictive ability}

We used the AGHmatrix package (Amadeu et al. 2016) to develop kinship G-matrices partitioning genetic variation based on several gene-action models. For the BT population DArTSeq markers (sweetpotato) where we did not have dosage information, we developed an additive G-matrix according to VanRaden (2008), herein referred to as Add_2x_DArTseq, and a non-additive-effects G-matrix according Vitezica et al. (2013), herein referred to as NonAdd_2x_DArTSeq. For the BT population GBSpoly (sweetpotato) and TON population GBSCornell (potato) data where we had dosage information, we employed three models to develop the G-matrices: (i) modeling only additive effects, according to VanRaden (2008) herein referred to as Add_6x_GBSpoly for sweetpotato and Add_4x_ GBSCornell for potato, (ii) modeling additive plus nonadditive effects, according to Slater et al. (2016) herein referred to as Add + Non_6x_GBSpoly for sweetpotato and Add + Non_4x_GBSCornell for potato and (iii) a pseudodiploidized effect model according to Slater et al. (2016), herein referred to as Pseudo_2x_GBSpoly for sweetpotato and Pseudo_2x_GBSCornell. The pseudo-diploidization collapses all dosage classes between the nulliplex and the hexaplex (in sweetpotato), and between the nulliplex and tetraplex (in potato) into one heterozygous class, under the assumption that all heterozygotes have an equal effect which falls in between both homozygotes. In the case of potato, the design matrix coding for the pseudo-diploid, additive autotetraploid and full autotetraploid was as 
described by Slater et al. (2016), while that for sweetpotato is shown in Table 3. During kinship matrix development, additional filters were applied to the genotype data, to have minimum allele frequency (MAF) $\geq 30 \%$, and call rate $\geq 90 \%$. We used genomic best linear unbiased prediction (G-BLUP; Clark and van der Werf 2013) to compare the predictive ability (PA) of the five models for sweetpotato and three models for potato using the kinship matrices as variance-covariance matrices to fit the compressed linear mixed model (Zhang et al. 2010) and estimate genomic best linear unbiased predictors (G-BLUPs). The software GAPIT (Lipka et al. 2012) was used in the G-BLUP prediction fitting the following general model:

$y=1_{n} \mu+Z u+e$

where $y=$ vector of phenotypic data, $l_{n}$ is the vector of ones, $\mu=$ population mean, $Z=$ the known design matrix for genotypes, $u=$ random genetic effects and $\sim N\left(0, \sigma_{a}^{2}\right.$ Kor $\left.\sigma_{a+n a}^{2} K\right)$ with $K=$ kinship matrix, $a=$ additive model, $n a=$ non-additive model, $e=$ vector of residuals $\sim N\left(0, \sigma_{e}^{2} I\right)$.

Cross-validation was done by randomly setting $20 \%$ of the population to missing phenotypes to be used as a validation set. We used 1000 iterations (replications) to estimate the predictive ability of the models using both simple/oligo traits (quality traits in sweetpotato, disease traits in potato) and complex traits (storage root or tuber yield and yield component traits in both), as defined in Table 1.

The PA was calculated as Pearson's correlation between the observed BLUPs and the genome estimated breeding values (GEBVs). Differences in PA among models per trait were tested using a simple one-way analysis of variance with models as factor. The correlation coefficients per replication were Fisher Z-transformed and means compared on these $\mathrm{Z}$ values using a one-way ANOVA with models as factor. The average PA was then obtained by back transforming the average of the $\mathrm{Z}$ values. Quantitative-genetic parameters were tested for the additive model with or without dosage by obtaining the additive genetic variation $\left(\sigma_{a}^{2}\right)$ and random residual effects $\left(\sigma_{e}^{2}\right)$ from the mixed linear model and calculating narrow-sense heritability $\left(h^{2}\right)$ for each trait as:

$h^{2}=\frac{\sigma_{a}^{2}}{\left(\sigma_{a}^{2}+\sigma_{e}^{2}\right)}$

Additionally, we calculated the estimated rate of genetic gains from genomic selection per additive model with or without dosage for each trait according to Oliveira et al. (2019) as:

$\Delta G G=\frac{\left(i * \sigma_{a} * P A\right)}{L}$

where $\Delta G G=$ rate of genetic gains, $i=$ selection intensity, $\sigma_{a}=$ square root of additive genetic variation, $P A=$ predictive ability and $L=$ length of breeding cycle, assuming $L=5$ for sweetpotato following the accelerated breeding scheme currently implemented (Mwanga et al. 2017), and $L=8$ for potato.

\section{How many markers are adequate for prediction?}

For the sweetpotato $F_{1}$ population, we used the original GBSpoly data, using different filtering criteria to end up with different number of markers. We used three criteria (i) total number of SNPs filtered at 10\% MAF and $\geq 90 \%$ call rate, (ii) total number of SNPs filtered at $30 \%$ MAF and $\geq 90 \%$ call rate (used in the analyses above) and (iii) a random sample of 15,000 SNPs from the total number of SNPs and filtered at $30 \%$ MAF and $\geq 90 \%$ call rate. In potato, the total number of SNPs was filtered using two criteria: (i) $30 \% \mathrm{MAF}$ and $\geq 90 \%$ call rate, (ii) $40 \% \mathrm{MAF}$ and $\geq 90 \%$ call rate. Predictions were carried out for all traits measured using these criteria. To separate the effects of allele frequency from the effects of number of markers on PA, we also used the original GBSpoly data in sweetpotato, filtered at constant MAF and randomly sampled different number of markers, which we used to compare PA in one quality-related simple trait ( $\beta$-carotene; $\mathrm{BC}$ ) and one yield-related complex trait (total number of storage roots;
Table 3 Proposed design matrix coding for auto-hexaploid sweetpotato as adapted from Slater et al. 2016

\begin{tabular}{llllllllll}
\hline Effects/marker & Pseudo_2x & Add_6x & \multicolumn{7}{l}{ Add+Non_6x } \\
\cline { 5 - 10 } & 1 & 1 & 1 & 2 & 3 & 4 & 5 & 6 & 7 \\
\hline AAAAAA & 0 & 0 & 1 & 0 & 0 & 0 & 0 & 0 & 0 \\
AAAAAB & 1 & 1 & 0 & 1 & 0 & 0 & 0 & 0 & 0 \\
AAAABB & 1 & 2 & 0 & 0 & 1 & 0 & 0 & 0 & 0 \\
AAABBB & 1 & 3 & 0 & 0 & 0 & 1 & 0 & 0 & 0 \\
AABBBB & 1 & 4 & 0 & 0 & 0 & 0 & 1 & 0 & 0 \\
ABBBBB & 1 & 5 & 0 & 0 & 0 & 0 & 0 & 1 & 0 \\
BBBBBB & 2 & 6 & 0 & 0 & 0 & 0 & 0 & 0 & 1 \\
\hline
\end{tabular}


RYTHA). We used 10, 000, 5000, 1000 and 500 SNPs, all filtered to $\mathrm{MAF} \geq 5 \%$. The model considering only additive effects (Add_6x_GBSpoly) was used in comparing the effect of allele frequency and number of markers in sweetpotato, while all three models were tested between the two filtering criteria in potato.

\section{Incorporating haplotypic-QTL in prediction models for sweetpotato}

By taking advantage of the fully phased integrated linkage map from BT (Mollinari et al. 2020), we tested the predictive ability from QTL-informed models. To achieve this, we used the same cross-validation scheme as above, where 80\%:20\% random samples were used as training and testing populations, respectively, replicated 1000 times. In order to detect QTL, we ran our random-effect multiple interval mapping (REMIM) using a sequential forward search (Pereira et al. 2020). Using the sequential forward search, we used score statistics to test map positions every 2 centiMorgans (cM) and added QTL to the random-effect model, one QTL at a time, using a relaxed genome-wide significance level threshold $(\alpha=0.20)$. A window size of $20 \mathrm{cM}$ was used to avoid selection of another position very close to a QTL already in the model. For G-BLUP models, realized kinship matrices were based on the haplotype information from markers positioned every $2 \mathrm{cM}$ in the genetic map. For QTL-BLUP (Q-BLUP), realized kinship matrices were based on the haplotypes from QTL-peak marker; if there were more than one QTL, their kinship matrices were averaged out; if there were no QTL, we obtained the prediction as in G-BLUP. For Q+ G-BLUP models, two terms were fitted, each with realized kinship matrices based on QTLpeak markers (like for Q-BLUP) and the remaining markers in the linkage map except those selected as QTL.

\section{Results}

\section{SNP profiles from the genotyping platforms}

DArTseq sequencing of sweetpotato resulted in 13,504 biallelic SNPs (Online Resource 3). The call rates and polymorphic information content (PIC) are shown in Fig. 1a, b and ranged from about $0.4(40 \%)-1.0(100 \%)$, with a mean of $0.96(96 \%)$ for call rate and from 0 to 0.5 with a mean of 0.37 for PIC. Stringent filtering at a call rate $\geq 80 \%$ and PIC $\geq 0.25$ left 9649 SNPs that were used in AGHMatrix. Additional
A

BT_DArTSeq CallRate

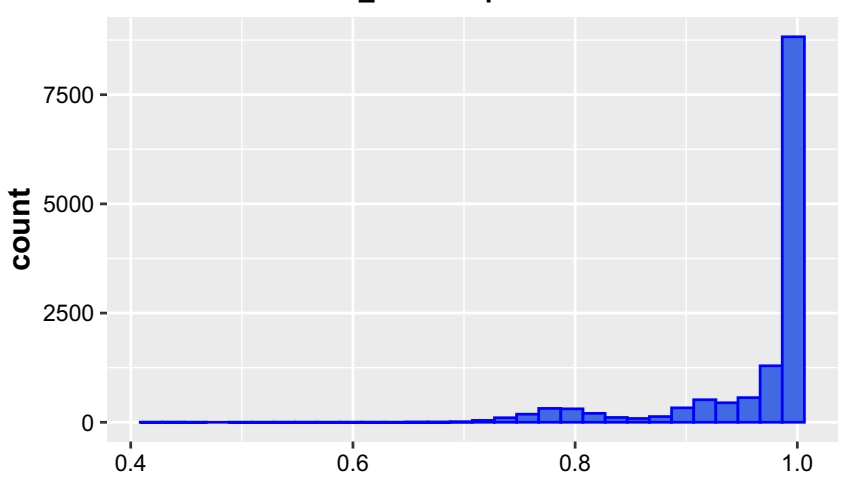

C

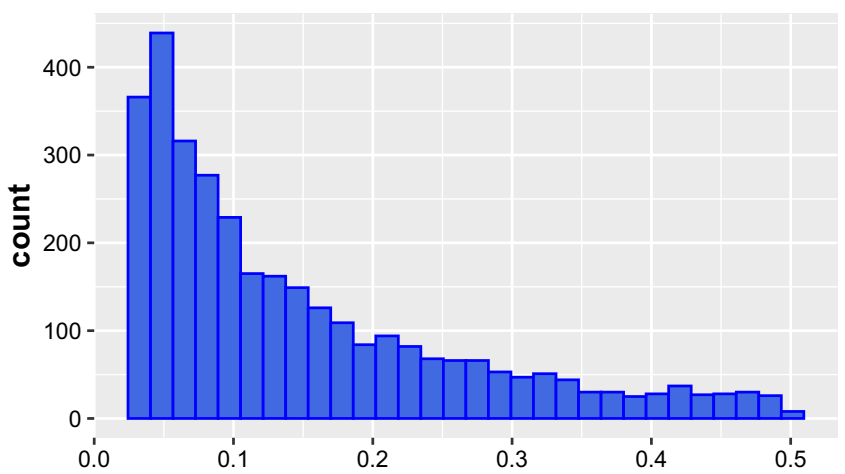

B

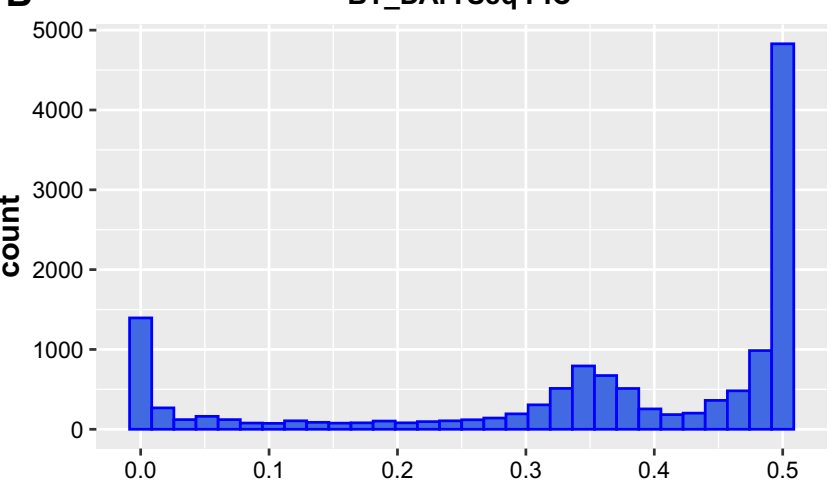

D

TON_GBSCornell PIC

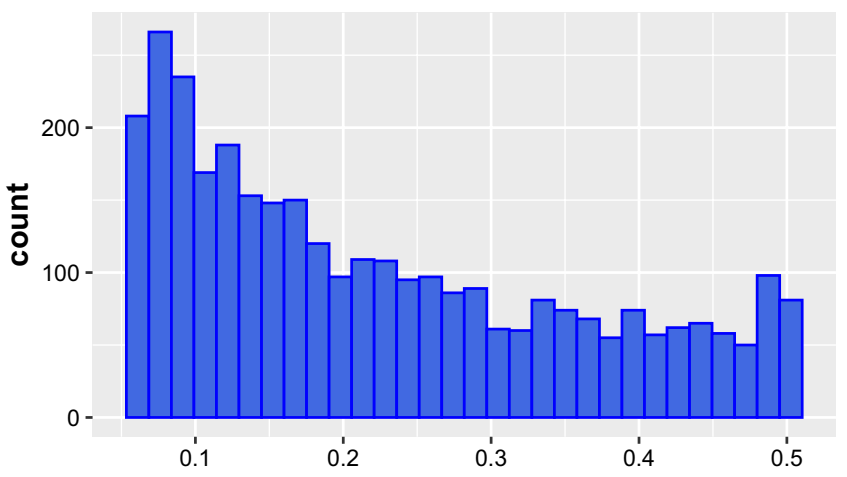

Fig. 1 Quality attributes of the SNP profiles from DArTSeq (call rate (a) and polymorphic information content; PIC (b)) data in sweetpotato and GBSCornell (minor allele frequency; MAF (c) and PIC (d)) in potato. The y-axes in all plots refer to the number of SNPs 
filtering in AGHMatrix at $\geq 80 \%$ call rate and $\geq 30 \%$ MAF resulted in 6015 diploidized, biallelic SNPs being used to develop the matrices following additive (Add_2x_DArTSeq) and non-additive (NonAdd_2x_DArTSeq) models.

Cornell GBS in potato resulted in 295,401 biallelic SNPs at the variant calling step that were then hard-filtered to 3262 high confidence SNPs by setting MAF $\geq 3 \%$, call rate $\geq 70 \%$ and average read depth (DP) $\geq 16$ (Online Resource 4). The 3262 SNP profiles are shown in Fig. 1C, D showing MAF ranging from $0.03(3 \%)$ to $0.5(50 \%)$, with a mean of 0.15 (15\%) and PIC ranging from 0.0 to 0.5 , with a mean of 0.23 . The 3262 SNPs were used in the AGHMatrix relationship matrix development. For a relative comparison of models across crops for trait groups, we also filtered the Cornell GBS data in AGHMatrix at $\geq 90 \%$ call rate and $\geq 30 \%$ MAF as done for DArTSeq data above, which resulted in 411 SNPs used to develop the additive (Add_4x_GBSCornell), additive plus non-additive (Add + Non_4x_GBSCornell) and the pseudo-diploidized (Pseudo_2x_GBSCornell) models. Examining the relationship matrices indicated that at MAF $\geq 30 \%$, the full model (Add + Non_4x_GBSCornell) was mainly monomorphic. For potato therefore, we also changed the MAF to $\geq 40 \%$, which resulted in 178 SNPs that were used to develop a second set of relationship matrices. All PA comparisons among traits for potato are based on this matrix (MAF $\geq 40 \%)$.

For GBSpoly in sweetpotato called according to Wadl et al. (2018), the empirical estimation of read depth threshold to ensure high-fidelity SNPs was carried out after paralog filtering (Fig. 2). The stability of each genotypic classes (nulliplex to hexaploid) was evaluated with increasing read depth, which was achieved by resampling reads (without replacement) to simulate Illumina sequencing of each locus at incremental read depths. SNPs derived from paralogs or repetitive sequences were eliminated due to low stability even at high read depths (more details in Wadl et al. 2018). Figure 3 shows the allele frequency of the GBSpoly data

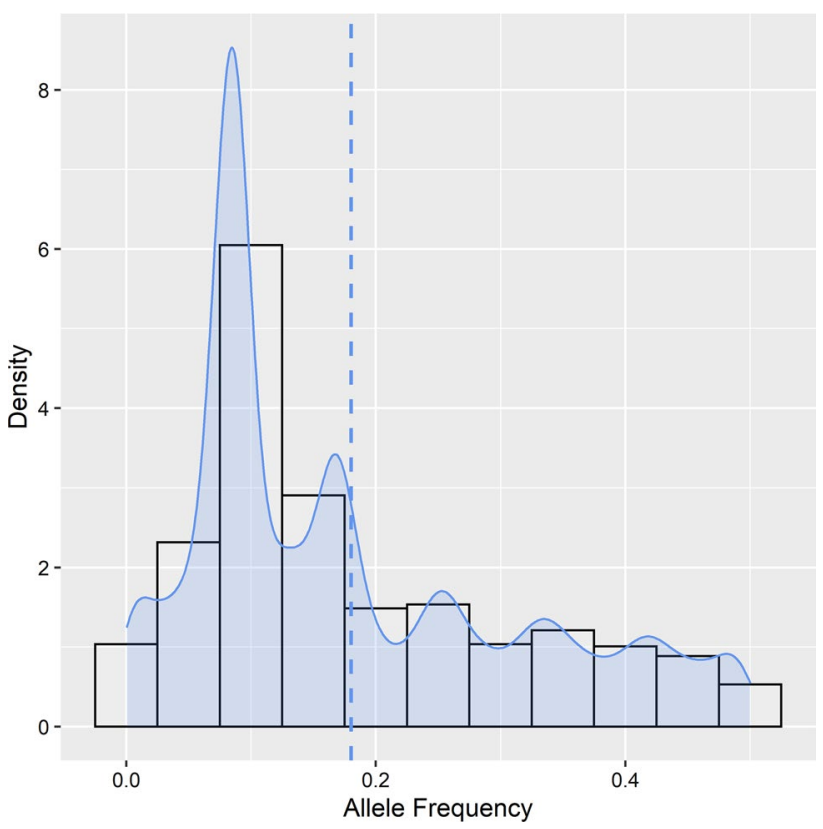

Fig. 3 Allele frequency density plot for GBSpoly data in sweetpotato

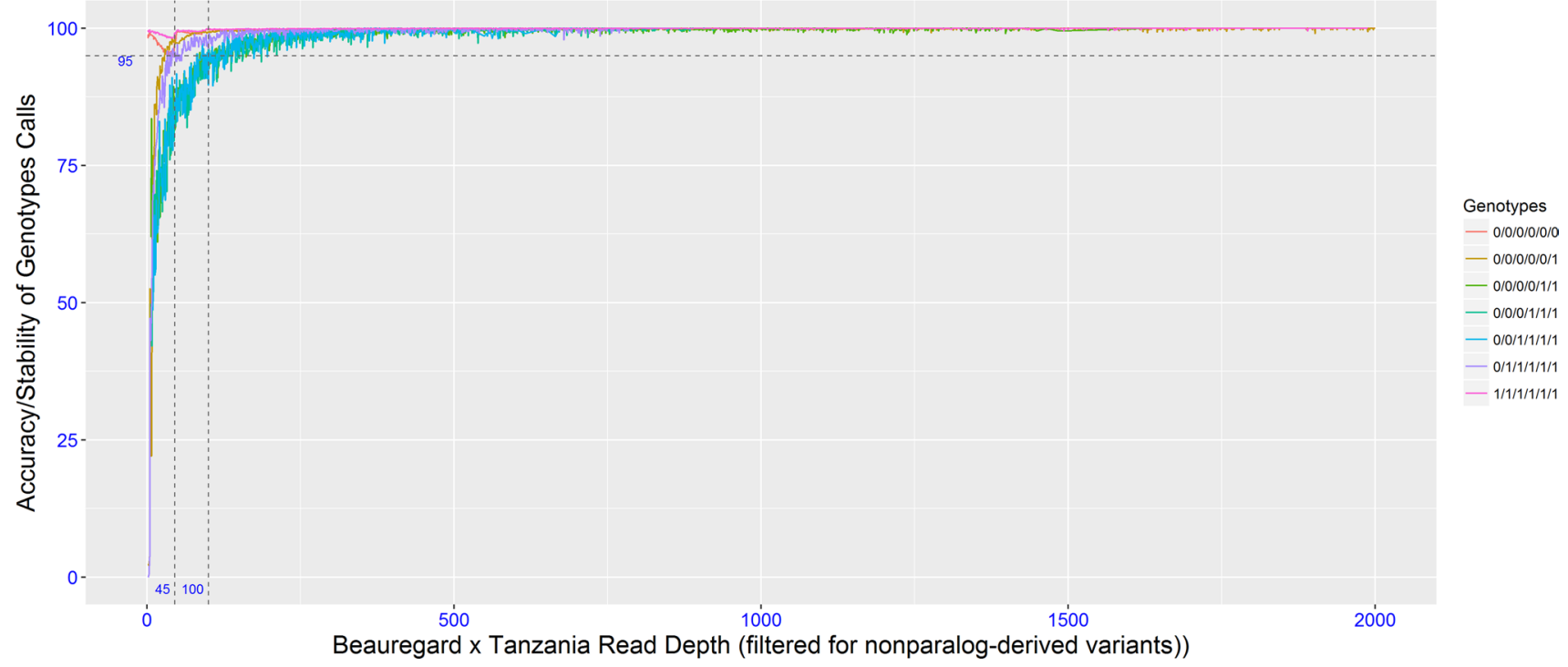

Fig. 2 Paralog filtering and empirical estimation of read depth threshold for high-fidelity SNPs in sweetpotato. The plot shows stability of each genotypic classes (nulliplex to hexaploid) with increasing read depth, which is achieved by resampling reads (without replacement) to simulate Illumina sequencing of each locus and at incremental read depths. SNPs derived from paralogs or repetitive sequences were eliminated due to low stability even at high read depths (more details in Wadl et al. 2018) 
ranging from 0 to $50 \%$, while expected segregation ratios in hexaploid sweetpotato as well as genotype distribution in each segregation class after filtering for segregation distortion at unmethylated loci are provided as Online Resource 5. Consequently, for sweetpotato, GBSpoly data were filtered to this high depth of coverage, with $\mathrm{MAF} \geq 5 \%$. This resulted in 34,390 high confidence SNPs (Online Resource 6) that were used in AGHMatrix to develop the additive (Add_6x_ GBSpoly), additive plus non-additive (Add + Non_6 GBSpoly) and the pseudo-diploidized (Pseudo_2x_GBSpoly) relationship matrices. The filters in AGHMatrix were set to $\geq 90 \%$ call rate and $\geq 30 \%$ MAF as for the preceding data types and resulted in a final 2883 SNPs that developed the matrices for model comparison. For comparing the combined effects of number of markers and allele frequency on PA, the first filtering criteria of 10\% MAF and $\geq 90 \%$ call rate resulted in 10,358 SNPs, while the third criteria based on a random sample of 15,000 SNPs resulted in 1291 SNPs at $\geq 30 \%$ MAF and $\geq 90 \%$ call rate that were used in PA comparison, based on the model considering additive effects only (Add_6x_GBSpoly). To examine the effect of markers only, without the compounding effect of allele frequency, 500, 1000, 5000 and 10,000 SNPs were randomly selected from the original 34,390 SNPs all at MAF $\geq 5 \%$ and tested using the model considering additive effects only (Add_6x_GBSpoly).

The comparison of models with(out) QTL and use of markers per se or haplotypes was carried out only in sweetpotato, using 30,684 SNPs from the same genotyping platform and data set, filtered and processed as described by Mollinari et al. (2020), which were used to develop a $2708.4 \mathrm{cM}$ phased genetic linkage map for sweetpotato, and subsequent QTL analyses (Pereira et al. 2020; Gemenet et al. 2020). The QTL summary tables in sweetpotato as previously reported by Pereira et al. (2020), Gemenet et al. (2020) are provided as Online Resource 7. Sweetpotato BLUEs are provided in Online Resource 8, while potato BLUEs are provided as Online Resource 9.

\section{Genotyping platforms, genetic effects and predictive ability}

In sweetpotato, the diploidized additive model (Add_2 DArTSeq) using data from DArTSeq performed equally well or sometimes better than the additive model using high confidence dosage data from GBSpoly (Add_6x_GBSpoly), depending on trait architecture, for simpler quality-related traits (Fig. 4). DM had 0.33 and 0.39, Starch had 0.32 and 0.34 , BC had 0.43 and 0.39 , FC_P had 0.44 and 0.42 , while FC_U had 0.42 and 0.35 average PA for Add_2x_DArTSeq and Add_6x_GBSpoly models, respectively (Table 4). For these traits, additive-only models were the best and the full model (Add_Non_6x_GBSpoly) always had negative PA due to a largely monomorphic relationship matrix, possibly due to the assumptions taken in calculating the additive and non-additive effects in the full model which may be complicated by the many possible combinations in autopolyploids. However, the situation changed with yield-related traits as the effects of dosage and non-additive effects became more important. For these traits, the high-quality data with dosage from GBSpoly (Add_6x_GBSpoly) were always better in prediction when compared to the additive model with diploidized data (Add_2x_DArTSeq). NOCR had 0.19 and 0.32 , TNR had 0.24 and 0.38 , CYTHA had 0.18 and 0.20 , RYTHA had 0.18 and 0.22 , and FYTHA had 0.21 and 0.24 average PA for Add_2x_DArTSeq and Add_6x_GBSpoly additive models, respectively (Table 4). However, the additive-only model with dosage (Add_6x_GBSpoly) was not always the best in PA for all yield-related traits, especially not for storage roots traits CYTHA and RYTHA, where it performed similar to either or both of the models considering non-additive effects whether with dosage (Add + Non_6x_ GBSpoly) or without dosage (NonAdd_2x_DArTseq) (Fig. 4). Nevertheless, the largely monomorphic relationship matrix from the full model (Add + Non_6x_GBSpoly) ensured low predictive ability using this model for most yield-related traits as well, especially FYTHA which had the highest negative PA, (collapsed to zero in Fig. 3, for plotting purposes). In general, pseudo-diploidizing high-quality data already called with dosage (Pseudo_2x_GBSpoly) drastically reduced PA even more than using data called as diploid (DArTseq). In potato, the situation was not very different as the pseudo-diploidized additive-effects model (Pseudo_2 GBSCornell) was the second-best model after the additiveeffects-only model with dosage (Add_4x_GBSCornell) for simpler disease traits and its comparative advantage significantly reduced with more complex traits (Fig. 5). LB2014_O had 0.68 and 0.63, LB2016_Y had 0.62 and 0.52, PVY_L had 0.55 and 0.51 , AYP_K had 0.45 and 0.34 , WMT_K had 0.48 and 0.34 , TTW16_Ica had 0.16 and 0.16 , while TTW16_HLJ had 0.38 and 0.31 average PA for Add_4 GBSCornell and Pseudo_2x_GBSCornell, respectively. As with the full model in sweetpotato, the model including nonadditive effects (Add + Non_4x_GBSCornell) was the least performing in terms of PA (Table 4).

\section{Number of markers and environments}

Our results in potato indicated that an increased number of markers by more than double did not have an effect of equal magnitude on PA (411 vs 178 SNPs; Fig. 5a vs b) considering the best predictive model (Add_4x_GBSCornell). LB2014_O had 0.69 and 0.68, LB2016_Y had 0.66 and 0.62, PVY_L had 0.59 and 0.55, AYP_K had 0.51 and 0.45 , WMT had 0.51 and 0.48 , TTW16_Ica had 0.19 and 0.16 , while TTW16_HLJ had 0.40 and 0.38 average PA for 
A

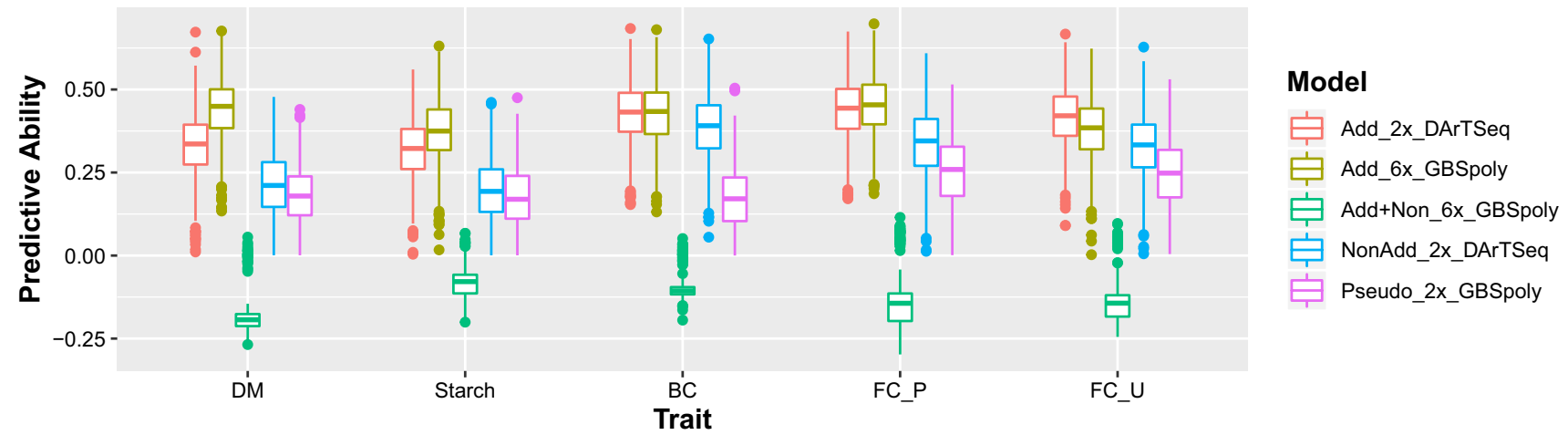

B

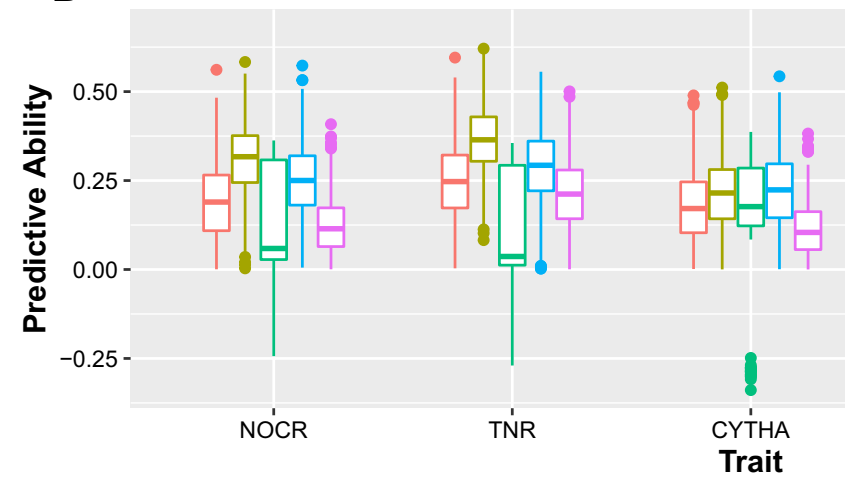

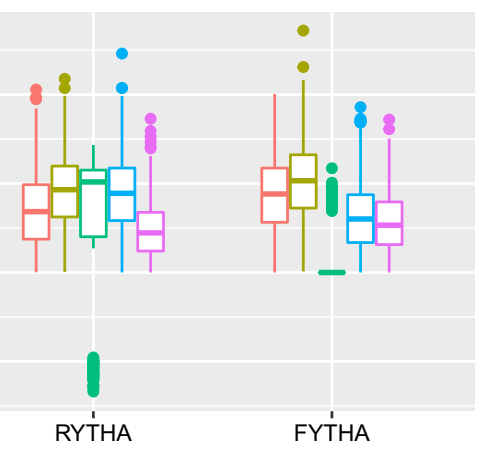

Model

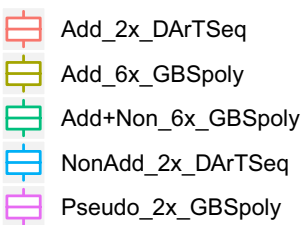

Fig. 4 Model comparison in sweetpotato. Boxplots comparing predictive ability of additive-effects-only models without dosage (Add_2x_DArTseq) and with dosage (Add_6x_GBSpoly); models considering also non-additive effects (NonAdd_2x_DArTSeq; Add + Non_6x_GBSpoly); and pseudo-diploidized dosage data (Pseudo_2x_GBSpoly) for quality-related traits (A; DM=dry mat-

411 and 178 SNPs, respectively. Similarly, in sweetpotato, comparing PA using 10,358 SNPs, 2883 SNPs and 1291 SNPs using the best predictive model (Add_6x_GBSpoly) showed no effect of increasing marker density at the cost of marker informativeness (allele frequency) on PA. PA based on 10,358 SNPs which had $\geq 10 \%$ MAF generally performed lower than 2883 and 1291 SNPs which both had $\geq 30 \%$ MAF (Fig. 6). Additionally, 2883 SNPs did not have a clear comparative advantage over 1291 SNPs, both at $\geq 30 \%$ (Fig. 6), indicating that allele frequency had more effect on PA than number of markers per se, and that less informative SNPs required a higher number of markers than more informative SNPS. This was confirmed in sweetpotato by using different numbers of SNPs randomly selected from the original SNP pool having a constant $\mathrm{MAF} \geq 5 \%$, where 10,000 and 5000 SNPs had better PA compared to 1000 and 500 SNPs for both simple (BC) and complex (RYTHA) traits (Online Resource 10). Regarding traits in different locations, environmental effects on PA were observed which can be attributed to genotype-by-environment interaction $(\mathrm{G} \times \mathrm{E})$. The PA based on the best model for FC_P $(0.44$; Peru $)$ and FC_U ter, starch, $\mathrm{BC}=\beta$-carotene, FC_P $=$ flesh color in Peru; FC_U $=$ flesh color in Uganda); and yield-related traits $(\mathrm{B}$; NOCR $=$ number of commercial storage roots, $\mathrm{TNR}=$ total number of storage roots, CYTHA = weight of commercial storage roots, RYTHA = weight of total storage roots, FYTHA = total weight of foliage) in a full-sib family of sweetpotato

(0.42; Uganda) in sweetpotato and LB2014_O (0.68; Peru) and LB2016_Y (0.62; China), TTW16_Ica (0.16; Peru) and TTW16_HLJ (0.38; China), in potato, were significantly different. However, the magnitude of the effect of $\mathrm{G} \times \mathrm{E}$ was higher for more complex yield trait, than for the simpler quality-related trait and disease trait. This can be attributed to the fact that complex traits are more prone to complex $\mathrm{G}$ $\mathrm{x} E$ interactions when compared to many simple traits.

\section{Effects of quantitative trait loci, haplotypes and dosage on predictive ability}

We additionally tested three analysis models using BT sweetpotato data: (i) Q-BLUP based on relationship matrices from QTL-peak haplotypes, (ii) Q + G-BLUP fitting two terms based on QTL-peak haplotypes and the rest of the markers in the linkage map, (iii) G-BLUP, predictions using markers spaced every $2 \mathrm{cM}$ in the genetic map without considering QTL. The PA results are shown in Fig. 7. Considering QTL haplotypes either per se (Q-BLUP) or with G-BLUP (Q + G-BLUP) had a clear comparative 
Table 4 Summary quantitativegenetic parameters derived from genomic selection with cross-validation applying different genetic effects models in sweetpotato and potato

\begin{tabular}{|c|c|c|c|c|c|c|c|}
\hline Crop & Trait $^{\mathrm{a}}$ & Model $^{\mathrm{b}}$ & $\sigma_{a}^{2}$ & $\sigma_{e}^{2}$ & $h^{2}$ & $P A$ & $\Delta G G$ \\
\hline \multirow[t]{20}{*}{ Sweetpotato } & \multirow[t]{2}{*}{ DM } & Add_2x_DArTSeq & 1.6935 & 2.9536 & 0.36 & $0.33 b$ & 0.085889 \\
\hline & & Add_6x_GBSpoly & 4.0035 & 2.0762 & 0.66 & $0.39 a$ & 0.176077 \\
\hline & \multirow[t]{2}{*}{ Starch } & Add_2x_DArTSeq & 6.1716 & 13.7616 & 0.31 & $0.32 b$ & 0.158993 \\
\hline & & Add_6x_GBSpoly & 12.1683 & 11.5424 & 0.53 & $0.34 \mathrm{a}$ & 0.265111 \\
\hline & \multirow[t]{2}{*}{$\mathrm{BC}$} & Add_2x_DArTSeq & 150.2336 & 113.1697 & 0.57 & $0.43 \mathrm{a}$ & 1.0541 \\
\hline & & Add_6x_GBSpoly & 225.1431 & 152.0581 & 0.60 & $0.39 b$ & 1.29041 \\
\hline & \multirow[t]{2}{*}{ FC_P } & Add_2x_DArTSeq & 0.5416 & 0.3304 & 0.62 & $0.44 \mathrm{a}$ & 0.064762 \\
\hline & & Add_6x_GBSpoly & 0.8168 & 0.4189 & 0.66 & $0.42 \mathrm{~b}$ & 0.081339 \\
\hline & \multirow[t]{2}{*}{ FC_U } & Add_2x_DArTSeq & 12.9633 & 10.9257 & 0.54 & $0.42 \mathrm{a}$ & 0.295238 \\
\hline & & Add_6x_GBSpoly & 16.1489 & 16.777 & 0.49 & $0.35 b$ & 0.305411 \\
\hline & \multirow[t]{2}{*}{ NOCR } & Add_2x_DArTSeq & 50134102 & $2.93 \mathrm{E}+08$ & 0.15 & $0.19 \mathrm{~b}$ & 269.0607 \\
\hline & & Add_6x_GBSpoly & $1.36 \mathrm{E}+08$ & $2.43 \mathrm{E}+08$ & 0.36 & $0.32 \mathrm{a}$ & 722.4699 \\
\hline & \multirow[t]{2}{*}{ TNR } & Add_2x_DArTSeq & $1.86 \mathrm{E}+08$ & $7.40 \mathrm{E}+08$ & 0.20 & $0.24 b$ & 681.9091 \\
\hline & & Add_6x_GBSpoly & $4.71 \mathrm{E}+08$ & $5.83 \mathrm{E}+08$ & 0.45 & $0.38 \mathrm{a}$ & 1606.149 \\
\hline & \multirow[t]{2}{*}{ CYTHA } & Add_2x_DArTSeq & 8.6149 & 27.7157 & 0.13 & $0.18 \mathrm{~b}$ & 0.105664 \\
\hline & & Add_6x_GBSpoly & 8.6149 & 26.6061 & 0.24 & $0.20 \mathrm{a}$ & 0.129145 \\
\hline & \multirow[t]{2}{*}{ RYTHA } & Add_2x_DArTSeq & 4.6249 & 31.4849 & 0.13 & $0.18 b$ & 0.07742 \\
\hline & & Add_6x_GBSpoly & 10.9811 & 29.4989 & 0.27 & $0.22 \mathrm{a}$ & 0.152434 \\
\hline & \multirow[t]{2}{*}{ FYTHA } & Add_2x_DArTSeq & 7.678 & 26.0083 & 0.23 & $0.21 b$ & 0.116379 \\
\hline & & Add_6x_GBSpoly & 12.8721 & 26.6023 & 0.33 & $0.24 \mathrm{a}$ & 0.186564 \\
\hline \multirow[t]{14}{*}{ Potato } & \multirow[t]{2}{*}{ LB2014_O } & Add_4x_GBSCornell & 0.0189 & 0.0193 & 0.49 & $0.68 \mathrm{a}$ & 0.011686 \\
\hline & & Pseudo_2x_GBSCornell & 0.0195 & 0.023 & 0.46 & $0.63 b$ & 0.010997 \\
\hline & \multirow[t]{2}{*}{ LB2016_Y } & Add_4x_GBSCornell & 0.0191 & 0.0259 & 0.42 & $0.62 \mathrm{a}$ & 0.010711 \\
\hline & & Pseudo_2x_GBSCornell & 0.0166 & 0.0323 & 0.34 & $0.52 b$ & 0.008375 \\
\hline & \multirow[t]{2}{*}{ PVY_L } & Add_4x_GBSCornell & 0.0419 & 0.0738 & 0.36 & $0.55 \mathrm{a}$ & 0.013817 \\
\hline & & Pseudo_2x_GBSCornell & 0.0364 & 0.0818 & 0.31 & $0.51 b$ & 0.011924 \\
\hline & \multirow[t]{2}{*}{ AYP_K } & Add_4x_GBSCornell & 0.0118 & 0.0327 & 0.27 & $0.45 \mathrm{a}$ & 0.00611 \\
\hline & & Pseudo_2x_GBSCornell & 0.0066 & 0.0389 & 0.15 & $0.34 b$ & 0.003453 \\
\hline & \multirow[t]{2}{*}{ WMT_K } & Add_4x_GBSCornell & 0.0132 & 0.0322 & 0.29 & $0.48 \mathrm{a}$ & 0.006893 \\
\hline & & Pseudo_2x_GBSCornell & 0.0069 & 0.0392 & 0.15 & $0.34 b$ & 0.00353 \\
\hline & \multirow[t]{2}{*}{ TTW16_Ica } & Add_4x_GBSCornell & $2.00 \mathrm{E}-04$ & 0.0028 & 0.07 & $0.16 \mathrm{a}$ & 0.000283 \\
\hline & & Pseudo_2x_GBSCornell & $3.00 \mathrm{E}-04$ & 0.0027 & 0.10 & $0.16 \mathrm{a}$ & 0.000346 \\
\hline & \multirow[t]{2}{*}{ TTW16_HLJ } & Add_4x_GBSCornell & 0.0061 & 0.018 & 0.25 & $0.38 \mathrm{a}$ & 0.003612 \\
\hline & & Pseudo_2x_GBSCornell & 0.0049 & 0.0192 & 0.20 & $0.31 \mathrm{~b}$ & 0.0028 \\
\hline
\end{tabular}

$\sigma_{a}^{2}$ is the additive genetic variation, $\sigma_{e}^{2}$ is the residual variance, $h^{2}$ is the narrow-sense heritability, $P A$ is the predictive ability, and $\Delta G G$ is the estimated rate of genetic gain considering the current breeding cycle length

${ }^{\text {a }}$ Traits as defined in Table 1

${ }^{b}$ Models: Add_2x_DArTseq = additive model using data from DArTseq called as diploid; Add_6x_GBSpoly =additive model using data with dosage from GBSpoly; Add_4x_GBSCornell=additive model using data with dosage from GBS at Cornell, Pseudo_2x_GBSCornell=additive model using data from GBSCornell with three heterozygote classes collapsed into one advantage for PA in simpler traits. However, this comparative advantage faded with more complex yield-related traits. Our results therefore show that with genomic selection, the comparative advantage of using the linkage map information and QTL is dependent on trait architecture, hence the magnitude of QTL effects that can be mapped (Fig. 7).

\section{Genetic variation, heritability and estimated rate of genetic gain}

Given that the additive-effects-only model with dosage performed better for most traits in both sweetpotato and potato (Add_6x_GBSpoly and Add_4x_GBSCornell, 
A

TON Potato traits_MAF0.3 411 SNPs

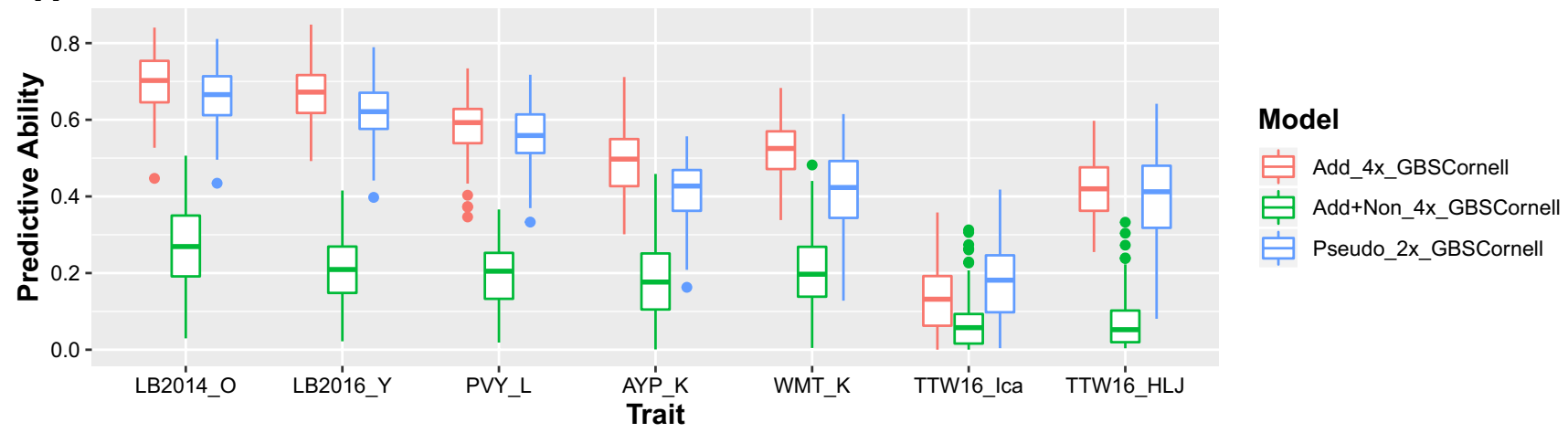

B

TON Potato traits_MAF0.4 178 SNPs

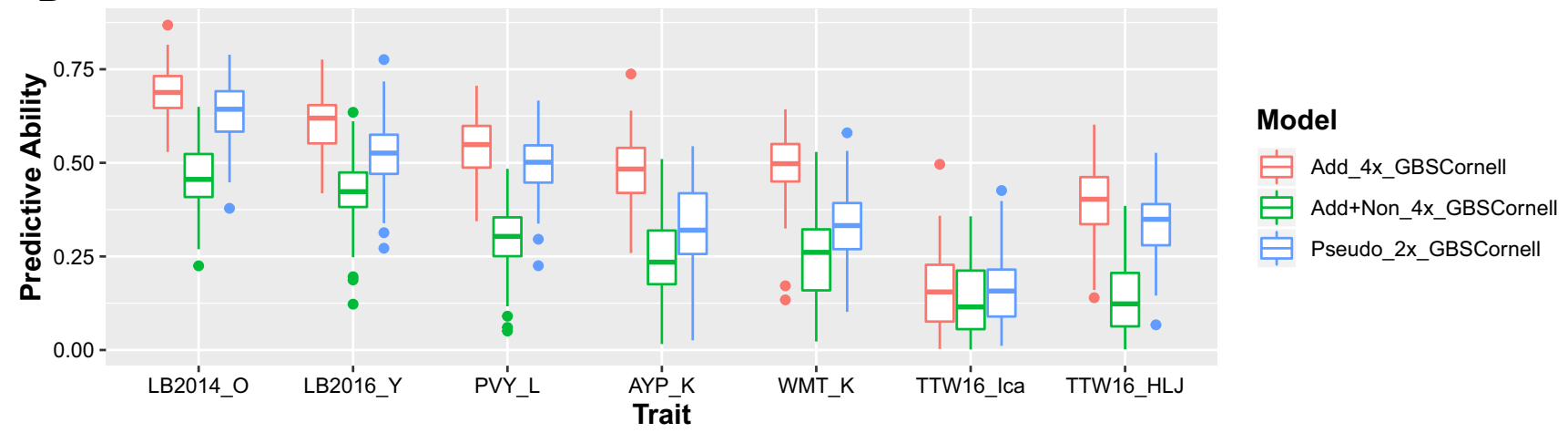

Fig. 5 Model comparison in potato. Box plots comparing predictive ability of additive-effects-only model (Add_4x_GBSCornell); additive and non-additive effects (Add + Non_4x_GBSCornell); and pseudo-diploidized dosage data (Pseudo_2x_GBSCornell); using minimum allele frequency (MAF) $\geq 30 \%$ (A; 411 SNPs) and $\mathrm{MAF} \geq 40 \%$ (B; 178 SNPs). LB2014_O=late blight in Oxapampa

respectively), we evaluated quantitative-genetic parameters for this model in comparison with the additive model without dosage for both crops (Add_2x_DArTseq for sweetpotato and Pseudo_6x_for potato). Narrow-sense heritability $\left(h^{2}\right)$ ranged from $0.24-0.66$ for the model with dosage (Add_6x_GBSpoly) and 0.13-0.62 for the model without dosage (Add_2x_DArTseq) in sweetpotato. In potato, $\left(h^{2}\right)$ ranged from 0.07 to 0.49 in the model with dosage (Add_4x_GBSCornell) and 0.10 to 0.46 in the model with pseudo-diploidized dosages (Pseudo2x_GBSCornell; Table 4). As expected, traits with simpler architecture (quality-related traits in sweetpotato; disease traits in potato) had the highest $\left(h^{2}\right)$ compared to more complex yield-related traits. All models across crops resulted in positive estimated genetic gain considering $L=5$ years in sweetpotato and $L=8$ in potato, which are the cycle lengths of current breeding schemes at CIP (Table 4). This implies that more genetic gains can be realized if such breeding cycle lengths are further significantly reduced.
(Peru) in 2014, LB2016_Y= late blight in Yunnan (China) in 2016, PVY_L = potato virus $Y$ in Lima (Peru), AYP_K= average yield per plant in Kunming (China), WMT_K=weight of marketable tubers in Kunming, TTW16_Ica = total tuber weight in Ica (Peru) in 2016 across three drought treatments, TTW16_HLJ = total tuber weight in Heilongjiang (China) in 2016, single treatment, in potato

\section{Discussion}

\section{Low-cost, targeted amplicon sequencing platforms could realize faster genetic gains per unit time}

Having a reliable, cost-efficient genotyping platform that ensures faster data turn-around to breeding programs on time to impact selection and advancement decisions is a must for routine application of genomic selection in plant breeding programs. Here we have compared results based on data from three GBS-based platforms, two of which provide data at the commercial diploid sequencing depth level (DArTSeq and GBSCornell). About $100 \times$ read depth was required to confidently call all the five heterozygous dosage classes of sweetpotato, against 25-30x required for the diploids. These results agree with studies in potato where Uitdewilligen et al. (2013) reported that 60-80x depth was required to confidently call the three heterozygote classes. GBSpoly (Wadl et al. 2018) provided high confidence, high density SNP data which are necessary for applications requiring high density markers such as genetic linkage mapping, QTL mapping 
A

Quality-related traits

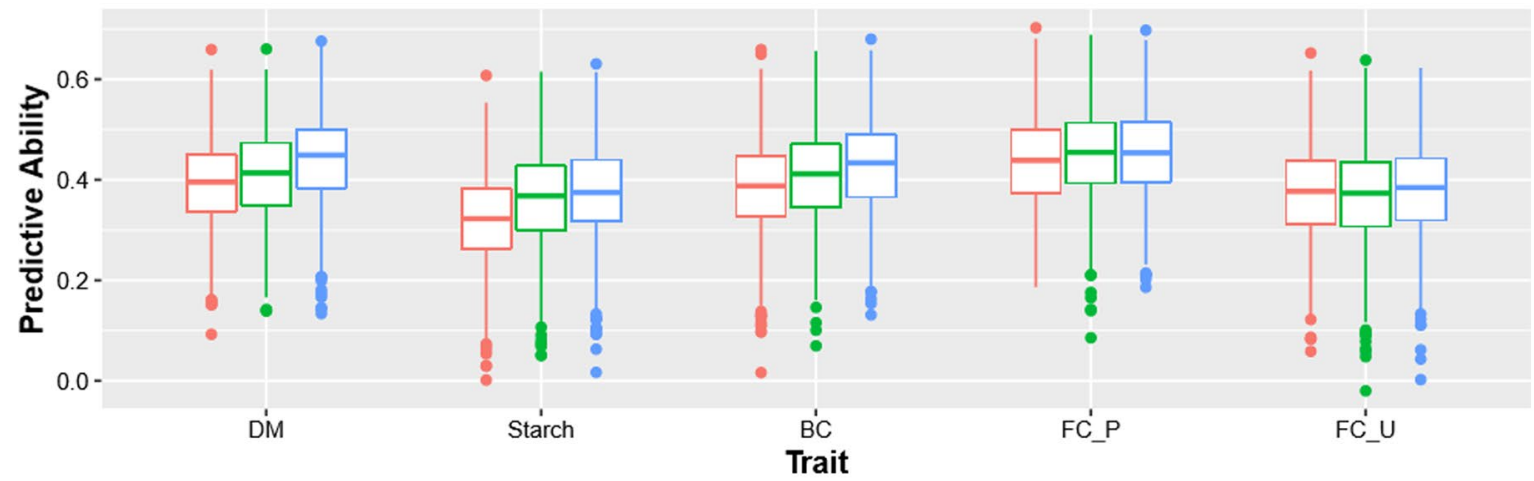

\#SNPS

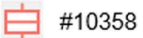

B

Yield-related traits

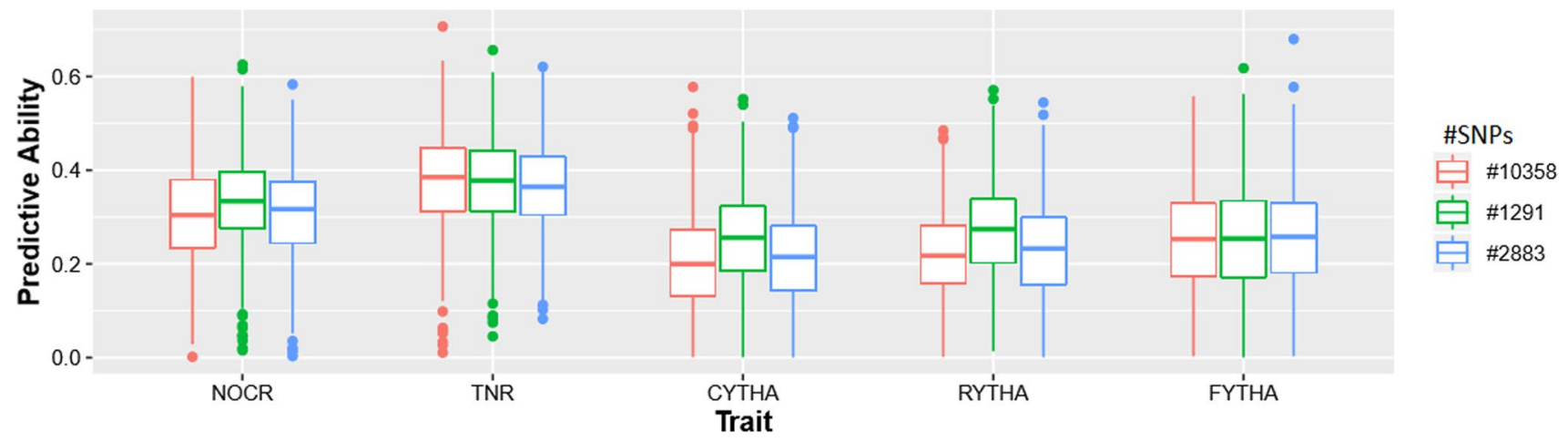

Fig. 6 Comparing effects of allele frequency and number of markers on predictive ability in sweetpotato. Box plots comparing the effect of number of markers (different minimum allele frequency) on predictive ability using additive-effects-only model (Add_6x_GBSpoly) with 10,358 SNPs, 2883 SNPs and 1291 SNPs in sweetpotato. $\mathrm{A} ; \mathrm{DM}=$ dry matter, starch, $\mathrm{BC}=\beta$-carotene, FC_P $=$ flesh color

and genome-wide association mapping for trait discovery pipelines. These resources are still limited in sweetpotato. However, its density and cost are currently not amenable to routine use in plant breeding, where resources are limited. Other options for more precise genotyping such as SNP arrays, in addition to issues with ascertainment biases, are crop-specific and therefore do not benefit from economies of scale that drive costs down. Breeding programs of polyploid crops therefore have to weigh whether investing more for higher depth of sequencing is an efficient resource allocation strategy (Endelman et al. 2018). To this end, although our results show that genotype quality and consequently the number of realized SNPs are lower with low allele sequencing depth, we also show that only a small number of markers are required to realize relatively high PA, if the SNPs are highly informative. It should, however, be noted that these results may apply only to the kind of populations analyzed in the current study, and that more complex populations like large, unstructured panels may require higher density markers. These results, however, agree with the findings of Chang et al. (2019) who showed that PA can be improved in Peru; FC_U =flesh color in Uganda; and yield-related traits: B; $\mathrm{NOCR}=$ number of commercial storage roots, $\mathrm{TNR}=$ total number of storage roots, CYTHA = weight of commercial storage roots, RYTHA = weight of total storage roots, FYTHA = total weight of foliage in a full-sib family of sweetpotato

by prioritizing relevant SNP polymorphisms. Similarly, Covarrubias-Pazaran et al. (2018) using three biparental populations of the American cranberry, showed that addition of SNPs after 500 markers did not result in much increase in PA as only a few hundred SNPs were needed to reach PA plateau. This therefore implies that for practical plant breeding applications, using established genotyping platforms that ensure low-costs due to scale effects and faster data turn-around will have better likelihood of success in routine application of genomic selection in polyploids. The costs associated with the need for high allele sequencing depth to confidently call SNPs could be offset by targeting only few but highly informative SNPs and investing a little more in sequencing depth of those. SNP informativeness is critical in this case, as our data show that allele frequency affects PA more than the number of markers. Guo et al. (2018) found that at allele sequencing depth between 10x and 20x, between 80 and $100 \mathrm{~K} \mathrm{SNPs}$ would be required to accurately predict additive breeding values in tetraploid rye grass. Our data similarly show that at low minimum allele frequency ( $\geq 5 \%$ ), 5000-10,000 SNPs had better PA than 500-1,000 
A
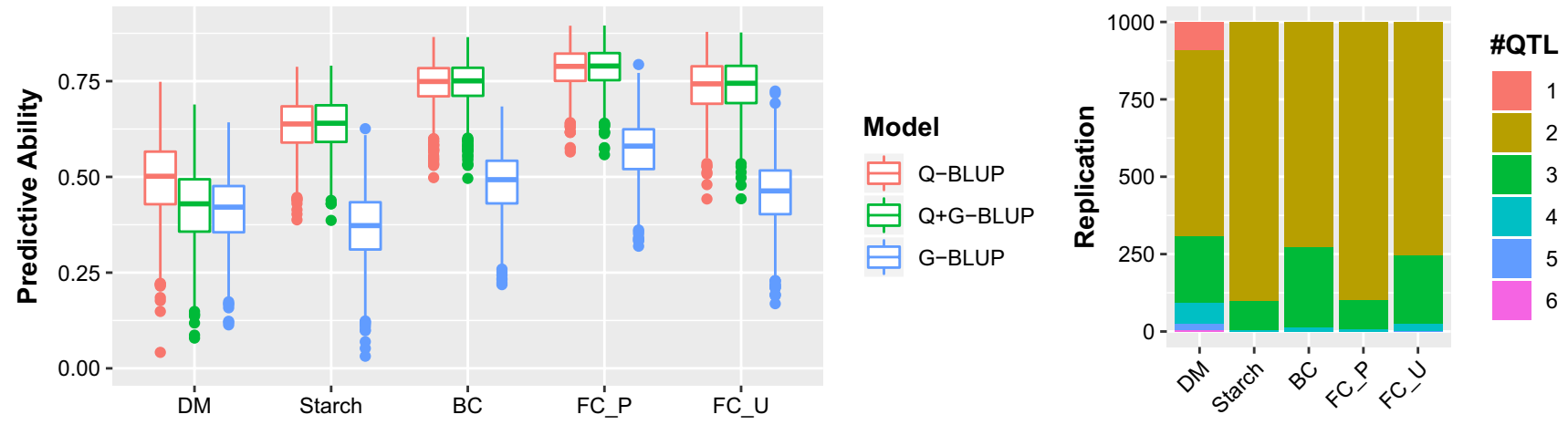

B

Yield-related traits
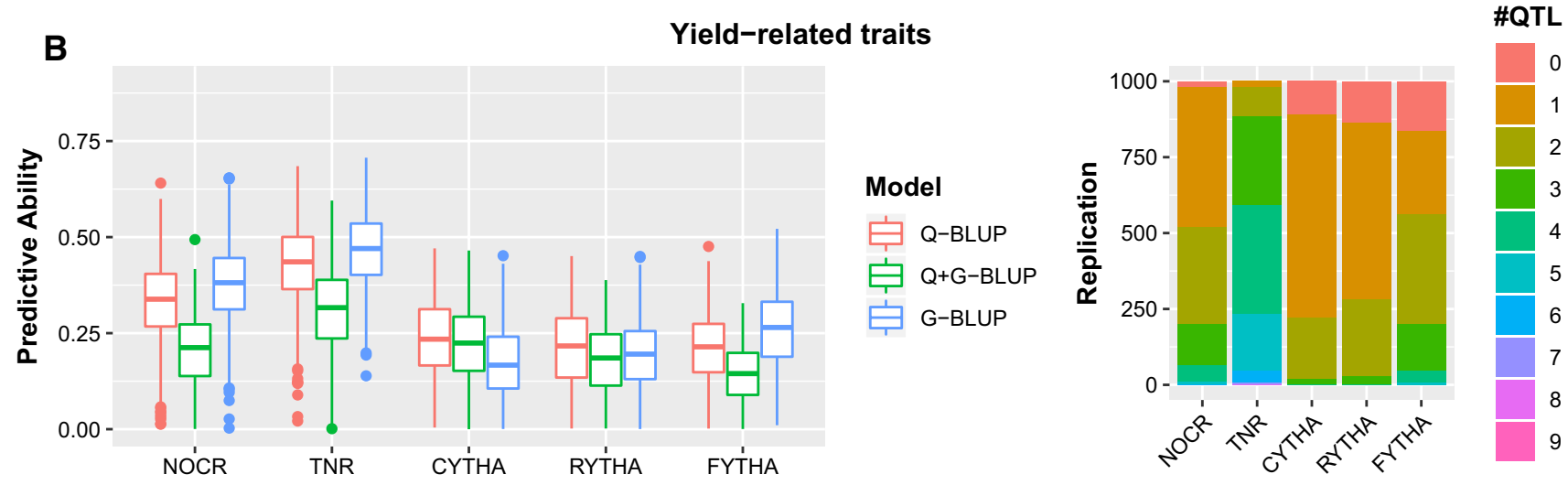

Fig.7 Effect of considering QTL haplotypes in sweetpotato. Boxplots comparing predictive ability of models using QTL haplotypes only in prediction (Q-BLUP); QTL combined with prediction based on markers per se, $(\mathrm{Q}+\mathrm{G}-\mathrm{BLUP})$; prediction using markers per se without QTL (G-BLUP) for quality-related traits (A; DM=dry matter, starch, $\mathrm{BC}=\beta$-caroten, FC_P $=$ flesh color in Peru; FC_U $=$ flesh color in Uganda); and yield-related traits $(B$; NOCR $=$ number of commercial storage roots, $\mathrm{TNR}=$ total number of storage roots, CYTHA = weight of commercial storage roots, RYTHA = weight of total storage roots, FYTHA = total weight of foliage) in a full-sib family of sweetpotato. The side graphs show the number of QTL in the model at a given replication (iteration) of cross-validation following the forward QTL search and backward QTL elimination used in the random-effect multiple interval mapping method
SNPs. Since both crops already have GBS-based SNPs at high density, the process can be fast-tracked by targeting the high informative segregating loci in amplicon sequencing. This is encouraging as polyploid crops in developing countries with limited access to expensive, high-quality genotypic datasets could also deploy GS approaches.

\section{Modeling non-additive genetic effects has negligible contribution to predictive ability}

Our results in both potato and sweetpotato show that additive-effects-only models, whether diploidized or with dosage, were comparatively better in PA than the models considering non-additive effects for all simple traits. This comparative advantage, however, lessened with more complex traits, where non-additive effects and inclusion of dosage information became slightly more relevant, although in most cases the additive-effects-only model with dosage still remained the best in terms of PA. This finding makes sense in quantitative genetic terms as the more the number of genes affecting a trait, the more the expected interaction among loci. In sweetpotato for example, issues of 'missing' heritability have been established for yield-related traits using the current BT population in multiple environments, where only a few QTL with very small effects were reported even though a very dense, well phased hexaploid genetic map was used (Pereira et al. 2020; Gemenet et al. 2020). According to Varona et al. (2018), the contribution of non-additive effects to genetic variance depends on the allele frequency of the causative loci, and their consideration in breeding programs can improve the prediction accuracy for breeding values and inform cross-combinations that maximize nonadditive variation in progeny. Several studies have, however, shown that inclusion of non-additive effects in the prediction models has negligible effects in improving the accuracy of predicting breeding (additive) values. For instance, Endelman et al. (2018) reported uncertainty in partitioning non-additive genetic variance in tetraploid potato, whereas Crow (2010), suggested that variance due to epistasis would have little effects in plant breeding as additive variance and 
covariance effects quickly overshadow such contribution following selection. Non-additive effects are mainly considered important in genomic prediction (prediction for performance of different traits based on the genotype of the individual), while additive-only methods as important in genomic selection (prediction of parental value of an individual), because only additive effects can be passed from parents to progeny (Varona et al. 2018). However, our results, supported by previous findings in other crops, imply that in light of the large number of moving parts to consider, including concerns with genotyping platforms and genotype quality for polyploids, practical breeding programs for potato and sweetpotato, and perhaps other polyploid crops, will achieve more advances considering only the infinitesimal model (additive) for both genomic selection and genomic prediction.

\section{The relative importance of considering dosage, haplotypes and quantitative trait loci is dependent on trait architecture}

Oliveira et al. (2019) showed that the relative advantage of including dosage information to PA is dependent on trait architecture. Our results confirm this and show that for simple traits diploidized data, especially when the genotypic data are directly called as diploid during variant calling, e.g., the DArTSeq data in sweetpotato rather than pseudo-diploidizing data already called with dosage, e.g., in GBSCornell data in potato, would be adequate for prediction. However, as the traits become more complex, considering dosage improves PA and therefore the rate of progress that can be made for such traits. Endelman et al. (2018) also showed that not considering allele dosage effects in potato reduced prediction accuracy by about 0.13 on average using data from the SolCAP potato SNP array, where they reported PA ranging from 0.06 to 0.63 for specific gravity, yield and fry color. Given that most traits are quantitative, we recommend the use of data with dosage that could benefit from improved genotype calling methods, such as Bayesian methods.

Our data in sweetpotato also show that for all traits, considering both QTL and haplotypes resulted in the best PA especially for simple traits, although this comparative advantage also faded with more complex yield traits. Having markers in complete LD with causative QTL for a given trait is a prerequisite for improving PA in genomic prediction (Velasco et al. 2019). The study of Cuyabano et al. (2014) showed that considering haplotype blocks rather than single markers improved PA for dairy traits in cattle. This is because haplotypes are supposed to be in tighter LD with QTL than single markers. This can be attributed to the fact that GS-only G-BLUP methods use the average genome information relationship for model building and for prediction whereas incorporating QTL analysis gives different weights (QTL effects) to different 'significant' genome positions (QTL positions) for model building and for prediction. Due to this, studies have proposed a combination of QTL mapping to explain trait architecture and genomic prediction, to improve PA (Spindel et al. 2016; Lopes et al. 2017; Morgante et al. 2018; Bhandari et al. 2019). Our results, however, indicate that the relative advantage of considering QTL-based haplotypes is dependent on trait architecture and directly related to the number and effect size of the QTL in question. In this case, yield-related traits did not show much improvement in PA when QTL were considered. Despite this finding, additional efforts in studying the effect of haplotype structure on PA are recommended to increase the likelihood of fully recovering the polyploid genetic information, where the information from individual dosage markers can be rather limited.

\section{Further considerations for optimized breeding programs using genomic selection}

The PA of genomic selection is influenced by several factors including trait architecture, the size of the training population, the relationship between the training and validation populations, heritability of the trait, the level of linkage disequilibrium (LD), marker density, environmental variances and covariance among traits (Nakaya and Isobe 2012). In addition to the already discussed factors, our results indicate that genotype-by-environment interaction plays a significant role in determining PA as can be seen in the same traits measured across several environments. Additionally, PA magnitude even for simple traits was lower in sweetpotato where we used BLUEs across six environments, than in potato where predictions were made per single environment. Models incorporating genotype- $x$-environment interaction are important and more realistic when predicting performance of untested genotypes across environments (Burgueno et al. 2012; Heslot et al. 2014; Wang et al. 2018). Furthermore, PA for complex yield-related traits was always lower than for simpler quality-related or disease traits. PA for such complex traits has been shown to benefit from multi-trait selection models incorporating simpler, correlated traits with the primary trait (Covarrubias-Pazaran et al. 2018; Michel et al. 2019). Additionally, Bernal-Vasquez et al. (2014) alluded to the fact that phenotypic data analysis contributed to improved PA, which speaks to the necessary precision and accuracy of the phenotype in training populations. Taken together, the current results show that genomic selection will contribute toward increased genetic gains, especially via reduced breeding cycle time in potato and sweetpotato. However, the effectiveness of genomic selection will have to be considered from the perspective of optimizing the entire breeding program (Cobb et al. 2019). Therefore, given the diversity existing from program to 
program in terms of resources and human capacity, no 'one size fits all' scenario is anticipated.

Finally, it does not escape to our attention that the predictions herein are based on single populations. However, plant breeding requires several levels of allele recombination through generations. We cannot estimate from the current data, how such recombination complexity will affect the efficiency of GS in breeding programs. Additional studies estimating PA in actual multi-generation breeding populations therefore need to be carried out to reliably estimate the value of GS to potato and sweetpotato, and perhaps other polyploid breeding programs. Furthermore, we used an $F_{l}$ population in sweetpotato and a fairly structured diversity panel in potato (Lindqvist-Kreuze et al. 2020), which may influence the number of markers required to carry out predictions. For routine application of GS therefore, there is need to determine the number of markers based on the population, its allele frequency and population structure.

Acknowledgements The sweetpotato research, most co-authors and GBSpoly genotyping were supported by a Bill \& Melinda Gates Foundation grant (Grant Number OPP1052983). DArTSeq genotyping of sweetpotato was done through the collaboration of the Integrated Genotyping Service and Support platform (IGSS) and DArT ${ }^{\mathrm{TM}}$ in Australia. Potato research and genotyping at Cornell was supported by a GIZ grant for the project 'Accelerating the Development of Early-MaturingAgile Potato for Food Security through a Trait Observation and Discovery Network.' The work was done as part of the Consultative Group on International Agricultural Research (CGIAR)-Research Program on Roots, Tubers and Bananas (RTB) which is supported by CGIAR Fund Donors (http://www.cgiar.org/about-us/our-funders/). Research teams at CIP and collaborating partners in China are greatly appreciated for providing phenotypic data.

Author contribution statement DCG, Z-BZ, GCY and HC led and managed different aspects of sweetpotato research, HLK and HC lead and managed different aspects of potato research, DCG, BDB, GSP, MM and HLK carried out data analysis, DCG wrote the manuscript, and all co-authors read, edited and approved the manuscript.

Data availability All single nucleotide polymorphism (SNP) data used in the current manuscript are provided with the manuscript as Online Resources 3-4, 6 while all best linear unbiased estimators (BLUEs) are provided as Online Resources 8 and 9.

\section{Compliance with ethical standards}

Conflict of interest On behalf of all co-authors, the lead author declares no conflict of interest.

Open Access This article is licensed under a Creative Commons Attribution 4.0 International License, which permits use, sharing, adaptation, distribution and reproduction in any medium or format, as long as you give appropriate credit to the original author(s) and the source, provide a link to the Creative Commons licence, and indicate if changes were made. The images or other third party material in this article are included in the article's Creative Commons licence, unless indicated otherwise in a credit line to the material. If material is not included in the article's Creative Commons licence and your intended use is not permitted by statutory regulation or exceeds the permitted use, you will need to obtain permission directly from the copyright holder. To view a copy of this licence, visit http://creativecommons.org/licenses/by/4.0/.

\section{References}

Altshuler D, Pollara VJ, Cowles CR, Van Etten WJ, Baldwin J et al (2000) An SNP map of the human genome generated by reduced representation shotgun sequencing. Nature 407:513-516

Amadeu RR, Cellon C, Olmstead JW, Garcia AA, Resende MF, Muñoz PR (2016) AGHmatrix: R package to construct relationship matrices for autotetraploid and diploid species: A blueberry example. Plant Genome. https://doi.org/10.3835/plantgenom e2016.01.0009

Baird NA, Etter PD, Atwood TS, Currey MC, Shiver AL et al (2008) Rapid SNP discovery and genetic mapping using sequenced RAD markers. PLoS ONE 3:e3376

Bernal-Vasquez A-M, Möhring J, Schmidt M, Schönleben M, Schön C-C, Piepho H-P (2014) The importance of phenotypic data analysis for genomic prediction - a case study comparing different spatial models in rye. BMC Genom 15:646

Bhandari A, Bartholome J, Cao- Hamadoun T-V, Kumari N, Frouin J, Kumar A et al (2019) Selection of trait-specific markers and multi-environment models improve genomic predictive ability in rice. PLoS ONE 14(5):e0208871

Blischak PD, Kubatko LS, Wolfe AD (2016) Accounting for genotype uncertainty in the estimation of allele frequencies in autopolyploids. Mol Ecol Resour 16:742-754

Blischak PD, Kubatko LS, Wolfe AD (2018) SNP genotyping and parameter estimation in polyploids using low-coverage sequencing data. Bioinformatics 34(3):407-415

Burgueño J, de los Campos G, Weigel K, Crossa J (2012) Genomic prediction of breeding values when modeling genotype $\mathrm{x}$ environment interaction using pedigree and dense molecular markers. Crop Sci 52:707-719

Chang L-Y, Toghiani S, Aggrey SE, Rekaya R (2019) Increasing accuracy of genomic selection in presence of high-density marker panels through the prioritization of relevant polymorphisms. BMC Genet 20:21

Clark SA, van der Werf J (2013) Genomic best linear unbiased prediction (gBLUP) for the estimation of breeding values. Methods Mol Biol 1019:321-330

Cobb JN, Juma RU, Biswas PS, Arbelaez JD, Rutkoski J, Atlin G, Hagen T, Quinn M, Ng EH (2019) Enhancing the rate of genetic gain in public-sector plant breeding programs: lessons from the breeder's equation. Theor Appl Genet 132:627-645

Courtois B, Audebert A, Dardou A, Roques S, Ghneim-Herrera T et al (2013) Genome-wide association mapping of root traits in a japonica rice panel. PLoS ONE 8(11):e78037

Covarrubias-Pazaran G, Schlautman B, Diaz-Garcia L, Grygleski E, Polashock J et al (2018) Multivariate GBLUP improves accuracy of genomic selection for yield and fruit weight in biparental populations of Vaccinium macrocarpon Ait. Front Plant Sci 9:1310

Crow JF (2010) On epistasis: why it is unimportant in polygenic directional selection. Philos Trans R Soc Lond B Biol Sci 365:1241-1244

Cruz VM, Kilian A, Dierig DA (2013) Development of DArT marker platforms and genetic diversity assessment of the US collection of the new oilseed crop lesquerella and related species. PLoS ONE 8(5):e64062

Cuyabano BCD, Su G, Lund MS (2014) Genomic prediction of genetic merit using LD-based haplotypes in the Nordic Holstein population. BMC Genom 15:1171 
De Silva HN, Hall AJ, Rikkerink E, McNeilage MA, Fraser LG (2005) Estimation of allele frequencies in polyploids under certain patterns of inheritance. Heredity 95:327-334

Elshire RJ, Glaubitz JC, Sun Q, Poland JA, Kawamoto K et al (2011) A robust, simple genotyping-by-sequencing (GBS) approach for high diversity species. PLoS ONE 6(5):e19379

Endelman JB, Carley CAS, Bethke BC, Coombs JJ, Clough ME et al (2018) Genetic variance partitioning and genome-wide prediction with allele dosage information in tetraploid potato. Genetics 209:77-87

Faville MJ et al (2018) Predictive ability of genomic selection models in a multi-population perennial ryegrass training set using genotyping-by-sequencing. Theor Appl Genet 131:703-720

Gemenet DC, Pereira GDS, De Boeck B, Wood JC, Mollinari M, Olukolu BA et al (2020) Quantitative trait loci and differential gene expression analyses reveal the genetic basis for negativelyassociated $\beta$-carotene and starch content in hexaploid sweetpotato [Ipomoea batatas (L.) Lam.]. Theor App Genet 133:23-36

Grüneberg W, Mwanga R, Andrade M, Espinoza J (2009) Breeding clonally propagated crops. In: FAO, selection methods: chapter 13 , part 5

Guo X, Cericola F, Fè D, Pedersen MG, Lenk I, Jensen CS, Jensen J, Janss LL (2018) Genomic prediction in tetraploid ryegrass using allele frequencies based on genotyping by sequencing. Front Plant Sci 9:1165

Heslot N, Akdemir D, Sorrells ME, Jannink JL (2014) Integrating environmental covariates and crop modeling into the genomic selection framework to predict genotype by environment interactions. Theor Appl Genet 127:463-480

Kilian A et al (2012) Diversity arrays technology: a generic genome profiling technology on open platforms. Methods Mol Biol 888:67-89

Lindqvist-Kreuze H, De Boeck B, Unger P, Gemenet D, Li X, Pan Z, Sui Q, Qin J, Woldegjorgis G, Negash K, Seid I, Hirut B, Gastelo M, De Vega J, Bonierbale M (2020) Global multi-environment resistance QTL for foliar late blight resistance in tetraploid potato with tropical adaptation. BioRxiv preprint. https://doi. org/10.1101/2020.02.16.950618

Lipka AE, Tian F, Wang Q, Peiffer J, Li M et al (2012) GAPIT: genome association and prediction integrated tool. Bioinformatics 28:2397-2399

Lopes MS, Bovenhuis H, van Son M, Nordbø $\varnothing$, Grindflek EH, Knol EF, Bastiaansen JWM (2017) Using markers with large effect in genetic and genomic predictions. J Animal Sci 95(1):59-71

Low JW, Mwanga ROM, Andrade M, Carey E, Ball A (2017) Tackling vitamin A deficiency with biofortified sweetpotato in sub-Saharan Africa. Global Food Secur 14:23-30

Meuwissen TH, Hayes BJ, Goddard ME (2001) Prediction of total genetic value using genome-wide dense marker maps. Genetics 157:1819-1829

Michel S, Löschenberger F, Ametz C, Pachler B, Sparry E, Bürstmayr H (2019) Simultaneous selection for grain yield and protein content in genomics-assisted wheat breeding. Theor Appl Genet 132: $1745-1760$

Mollinari M, Olokulu B, Pereira GDS, Khan A, Gemenet DC, Yencho C, Zeng Z-B (2020) Unraveling the hexaploid sweetpotato inheritance using ultra-dense multilocus mapping. G3: Genes, Genomes, Genetics 10(1):281-292

Morgante F, Huang W, Maltecca C, Mackay TFC (2018) Effect of genetic architecture on the prediction accuracy of quantitative traits in samples of unrelated individuals. Heredity 120:500-514

Mwanga ROM, Andrade MI, Carey EM, Low JW, Yencho GC, Grüneberg WJ (2017) Sweetpotato (Ipomoea batatas L.). In: Campos H, Caligari PDS (eds) Genetic improvement of tropical crops. Springer, Berlin, pp 181-218
Nakaya A, Isobe N (2012) Will genomic selection be a practical method for plant breeding? Ann Bot 110(6):1303-1316

Nyine M, Uwimana B, Blavet N, Hřibová E, Vanrespaille H, Batte M, Akech V, Brown A, Lorenzen J, Swennen R, Doležel J (2018) Genomic prediction in a multiploid crop: genotype by environment interaction and allele dosage effects on predictive ability in banana. Plant Genome 11:170090

Oliveira IDB, Resende MFR Jr, Ferrão LFV, Amadeu RR, Endelman JB, Matias Kirst M, Coelho ASG, Munoz PR (2019) Genomic prediction of autotetraploids; influence of relationship matrices, allele dosage and continuous genotype calls in phenotype prediction. Genes Genomes Genetics 9:1189-1198

Pereira GDS, Gemenet DC, Mollinari M, Olukolu BA, Diaz F, Mosquera V, Gruneberg WJ, Khan A, Yencho GC, Zeng Z-B (2020) Multiple QTL mapping in autopolyploids: a random-effect model approach with application in a hexaploid sweetpotato full-sib population. Genetics. https://doi.org/10.1534/genetics.120.303080

Piepho HP (1998) Empirical best linear unbiased prediction in cultivar trials using factor-analytic variance-covariance structures. Theor Appl Genet 97:195-201

Poland JA, Rife TW (2012) Genotyping-by-sequencing for plant breeding and genetics. Plant Genome J 5:92-102

Poplin R, Ruano-Rubio V, DePristo MA, Fennell TJ, Carneiro MO, Van der Auwera GA, Kling DE et al (2017) Scaling accurate genetic variant discovery to tens of thousands of samples. BioRxiv Preprint. https://doi.org/10.1101/201178

Raman H, Raman R, Kilian A, Detering F, Carling J et al (2014) Genome-wide delineation of natural variation for pod shatter resistance in Brassica napus. PLoS ONE 9(7):e101673

Slater AT et al (2016) Improving genetic gain with genomic selection in autotetraploid potato. Plant Genom 9(3):1-15

Spindel JE, Begum H, Akdemir D, Collard B, Redoña E, Jannink J-L, McCouch S (2016) Genome-wide prediction models that incorporate de novo GWAS are a powerful new tool for tropical rice improvement. Heredity 116:395-408

Uitdewilligen JG, Wolters AM, D'hoop BB, Borm TJ, Visser RG et al (2013) A next-generation sequencing method for genotyping-bysequencing of highly heterozygous autotetraploid potato. PLoS ONE 8:e62355

VanRaden PM (2008) Efficient methods to compute genomic predictions. J Dairy Sci 91(11):4414-4423

Varona L, Legarra A, Toro MA, Vitezica ZG (2018) Non-additive effects in genomic selection. Front Genet 9:78

Velazco JG, Malosetti M, Hunt CH, Mace ES, Jordan DR, van Eeuwijk FA (2019) Combining pedigree and genomic information to improve prediction quality: an example in sorghum. Theor Appl Genet 132:2055-2067

Vitezica ZG et al (2013) On the additive and dominant variance and covariance of individuals within the genomic selection scope. Genetics 195:1223-1230

Wadl PA, Olukolu BA, Branham SE, Jarret RL, Yencho GC et al (2018) Genetic Diversity and Population Structure of the USDA Sweetpotato (Ipomoea batatas) Germplasm Collections Using GBSpoly. Front Plant Sci 9:1-13

Wang X, Xu Y, Hu Z, Xu C (2018) Genomic selection methods for crop improvement: current status and prospects. Crop J 6:330-340

Watson A, Ghosh S, Williams M et al (2018) Speed breeding is a powerful tool to accelerate crop research and breeding. Nature Plants 4:23-29

Wu S, Lau KH, Cao Q, Hamilton JP, Sun H, Zhou C et al (2018) Genome sequences of two diploid wild relatives of cultivated sweetpotato reveal targets for genetic improvement. Nature communications 9:4580

$\mathrm{Xu}$ Y, Crouch JH (2008) Marker-assisted selection in plant breeding: from publications to practice. Crop Sci 48:391-407 
Zhang Z, Ersoz E, Lai CQ, Todhunter RJ, Tiwari HK et al (2010) Mixed linear model approach adapted for genome-wide association studies. Nat Genet 42:355-360
Publisher's Note Springer Nature remains neutral with regard to jurisdictional claims in published maps and institutional affiliations. 